\title{
Structural Reliability Sensitivities under Nonstationary Random Vibrations
}

\author{
Rita Greco $^{1}$ and Francesco Trentadue ${ }^{2}$ \\ ${ }^{1}$ DICATECh-Department of Civil, Environmental, Territory, Building and Chemical Engineering, \\ Technical University of Bari, Via Orabona, 4-70125 Bari, Italy \\ ${ }^{2}$ DICA-Department of Civil Engineering and Architectural Science, Technical University of Bari, Via Orabona, \\ 10-70125 Bari, Italy
}

Correspondence should be addressed to Rita Greco; r.greco@poliba.it

Received 31 July 2012; Revised 13 January 2013; Accepted 27 January 2013

Academic Editor: Erwei Bai

Copyright (C) 2013 R. Greco and F. Trentadue. This is an open access article distributed under the Creative Commons Attribution License, which permits unrestricted use, distribution, and reproduction in any medium, provided the original work is properly cited.

\begin{abstract}
Response sensitivity evaluation is an important element in reliability evaluation and design optimization of structural systems. It has been widely studied under static and dynamic forcing conditions with deterministic input data. In this paper, structural response and reliability sensitivities are determined by means of the time domain covariance analysis in both classically and nonclassically damped linear structural systems. A time integration scheme is proposed for covariance sensitivity. A modulated, filtered, white noise input process is adopted to model the stochastic nonstationary loads. The method allows for the evaluation of sensitivity statistics of different quantities of dynamic response with respect to structural parameters. Finally, numerical examples are presented regarding a multistorey shear frame building.
\end{abstract}

\section{Introduction}

The problem of structural response to dynamic nondeterministic actions has to be posed when structures, such as rotating machinery components or constructions subject to sea/wind loads, are designed to operate in random vibration environments. In these cases, due to the intrinsic random nature of inputs, a random dynamic analysis is needed, as documented in random vibration theory (e.g., [1-5]). Random dynamic analysis offers valid information about structural response otherwise impossible to obtain through classic deterministic analysis. A suitable evaluation of structural integrity, fundamental to design, can be obtained through this approach, in which structural safety is evaluated by the probability that the analyzed structure will not collapse during its working or life time. More specifically, this probability regards one or more components of a given structure and the critical state or conditions of failure these components reach in a given time interval. In this work, failure is related to a first crossing threshold, so that the structural crisis is associated with a first overcoming of one or more structural response measures (from a safe domain). Typically, structural response measures are displacements, stresses, buckling loads or natural frequencies. Moreover, statistics of response and structural reliability including their sensitivities (i.e., evaluation of partial derivatives of a performance measure with respect to system parameters), allows us to evaluate structural performances when some design variables have parametric changes. In particular, sensitivity analysis is a suitable way to evaluate the response variation of structures and, due to its importance in structural design, it is actually an important research topic in the field of computational mechanics. The sensitivities of structural responses are a fundamental information for gradient-based optimization methods needed in structural reliability analysis, optimization, and identification. Even random structural analysis with uncertain system parameters, obtained by removing commonly used deterministic assumptions (often extremely unrealistic), needs structural sensitivities. The analysis and design sensitivity computations do not offer quite hard difficulties for static problems, and 
analytical formulations for design sensitivity analysis are being reported in the literature from early seventies [68]. In dynamic problems, sensitivity analysis of structural response under transient dynamic loads has been studied especially in case of deterministic dynamic loads. Conversely not much research [9-11] seems to have been carried out for computation of design sensitivity under stochastic dynamic environment. Recently, an interesting analytical approach has been proposed by Chaudhuri and Chakraborty [12]. It is based on response sensitivity evaluation of structures in seismic reliability evaluation. The formulation has been developed in double frequency domain to take nonstationary earthquake motion for obtaining the analytical sensitivity statistics of various dynamic response quantities with respect to structural parameters. A semianalytical approach to evaluate the sensitivity of the stochastic response of both classically and nonclassically damped structural systems under stationary or nonstationary stochastic Gaussian excitation has been proposed [13]. The equations governing the evolution of the sensitivity of the first two statistical moments of the response for multi-degree-of-freedom $(\mathrm{MDoF})$ structural systems are first derived in analytical form in the time domain, once the Kronecker algebra, the series, expansions, and a suitable modal expansion of the deterministic response are adopted.

Finally in [14] a new closed-form analytical approximation to the first-passage problem is presented in structural reliability by using the exact closed-form solutions for the spectral characteristics of nonstationary random processes.

The main objective of the present work is to propose an analytical method to evaluate in time domain response covariance and reliability sensitivity for general linear structures subjected to nonstationary stochastic loads. This analytical approach presents a serious reduction in computational cost, especially if compared with numerical procedures. The evolutive random input is modelled by a nonstationary modulated filtered white noise process, able to represent many real physic loads, as the earthquake loads here considered. In order to obtain a general nonstationary approach to be used in different contexts, the structural response is evaluated by a covariance approach. By knowledge of evolutionary covariance matrix in state-space, the reliability is derived with reference to first crossing failure events. Sensitivity of covariance matrix and reliability is then obtained. Therefore, the proposed formulation computes the sensitivity gradient of covariance matrix and structural reliability with respect to the structural design parameters. A time integration algorithm for solving this problem is proposed, and finally a multistory building idealized by a shear frame structure (Figure 2) is considered to study the sensitivity of dynamic responses. The rigid floors masses and interstorey lateral stiffness and damping are considered as design variables.

\section{Linear Elastic MDoF Subject to Nonstationary Random Vibration}

Many real cases of structural problem deal with structural configurations that could be represented by linear viscoelastic lumped masses system, subject to stationary or nonstationary actions. In this work, both of these input conditions are considered to evaluate structural response statistics by means of the covariance approach. This offers serious advantages both in stationary and more in nonstationary conditions if inputs are modelled as white noises, eventually filtered to increase their accordance with real dynamic loads.

The structural response of a deterministic second order $m_{s}$-degree of freedom linear mechanical system subject to a probabilistic dynamic input is determined by solving the dynamic equilibrium system equations:

$$
\mathbf{M} \bar{X}_{s}(t)+\mathbf{C} \overline{\dot{X}}_{s}(t)+\mathbf{K} \overline{X_{s}}(t)=\mathbf{G}_{\mathrm{s}} \bar{f}(t),
$$

where $\overline{X_{s}}, \overline{\dot{X}_{s}}$, and $\bar{X}_{s}$ are the structural displacement, velocity, and acceleration process vectors, and $\mathbf{M}, \mathbf{C}$, and $\mathbf{K}$ are the mass, viscous and stiffness matrices.

The forcing vector

$$
\bar{f}(t)^{T}=\left[f_{1}(t), f_{2}(t), \ldots, f_{n}(t)\right]
$$

collects $n$ stochastic excitations applied to the structure, and $\mathbf{G}_{\mathrm{s}}$ is a $m_{s} x n$ matrix coupling the excitation components of forcing vector to the structural degrees of freedom. If system excitations vector elements are white noises, the first and second order statistical moments are time invariant, so that

$$
\begin{gathered}
\left\langle f_{i}^{S T}(t)\right\rangle=\mu_{f_{i}}, \\
\left\langle f_{i}^{S T}\left(t_{1}\right) f_{j}^{S T}\left(t_{2}\right)\right\rangle=[\mathbf{J}]_{i j} \delta\left(t_{2}-t_{1}\right)=\left[\mathbf{R}_{f f}^{S T}\left(t_{2}, t_{2}\right)\right]_{i j},
\end{gathered}
$$

where $\langle\cdot\rangle$ is for the mathematical expectation and $\delta(\cdot)$ is Dirac's delta function.

If $f_{i}^{S T}(t)$ are Gaussian excitations, the responses $X_{i}(t)$ and their time derivatives $\dot{X}_{i}(t)$ constitute a Markov vector in the $2 m_{s}$ dimension phase state.

The matrix $\mathbf{J}$, related to a vector satisfying the shot noise properties (usually denoted as a shot noise vector), has diagonal elements $[\mathbf{J}]_{i i}=2 \pi S_{0}^{i}$ equal to the auto covariance intensity of each force and extra diagonal elements equal to $[J]_{i j}=2 \pi S_{0}^{i j}=2 \pi S_{0}^{j i}$, representing the level of correlation between two generic different forces, so that $[\mathbf{J}]_{i j}$ can vary from $2 \pi S_{0}$, if $f_{i}$ and $f_{j}$ are completely correlated, to zero, if $f_{i}$ and $f_{j}$ are completely uncorrelated.

Then, in case of complete uncorrelated forcing loads, the matrix $\mathbf{J}$ is replaced by the more simple diagonal matrix of components $[\mathbf{D}]_{i i}=2 \pi S_{0}^{i}$, where the elements $S_{0}^{i}$ are the input power spectral density of each entry.

A widely adopted approach consists in representing a nonstationary input by means of an intensity modulation of a stationary process, also denoted as uniform modulation, in which it is assumed that process intensity changes during time as a deterministic function $\varphi(t)$, without any variation in spectral contents. Thus, in case of time modulation, a stationary forcing process vector is replaced by the following nonstationary vector:

$$
\bar{f}^{N S}(t)=\left[\varphi_{1}(t) f_{1}^{S T}(t), \varphi_{2}(t) f_{3}^{S T}(t), \ldots, \varphi_{n}(t) f_{n}^{S T}(t)\right]
$$


with the stochastic characterization

$$
\left\langle f_{i}^{N S}(t)\right\rangle=\varphi_{i}(t) \mu_{f_{i}}
$$

and, in case of uncorrelated excitations, the covariance matrix $\mathbf{R}_{f f}^{N S}\left(t_{2}, t_{2}\right)$ is a diagonal

$$
\begin{aligned}
\mathbf{R}_{f f}^{N S}\left(t_{1}, t_{2}\right) & =\left\langle f_{i}^{N S}\left(t_{1}\right) f_{j}^{N S}\left(t_{2}\right)\right\rangle \\
& = \begin{cases}2 \pi S_{0}^{i} \varphi_{i}\left(t_{1}\right) \varphi_{i}\left(t_{2}\right) \delta\left(t_{2}-t_{1}\right) & \text { if } i=j, \\
0 & \text { if } i \neq j .\end{cases}
\end{aligned}
$$

2.1. Prefilters Technique. The prefilter approach is adopted when the autocorrelation function of the input is not known. In this approach, input processes are modelled by solving filter differential equations, whose input is a white noise process. So doing, it is possible to give a representation of physical phenomena whose frequency characterization is subject to seriously variations in time, potentially causing structural resonant consequences, while maintaining the advantages offered by a shot noise input.

In particular, in the prefilter approach, the filter response is described by the $2 m_{f}$ filter state-space vector $\bar{Z}_{f}=$ $\left(\bar{X}_{f}, \bar{X}_{f}\right)^{T}$, solution of the $2 m_{f}$ set of differential equations

$$
\bar{Z}_{f}=\left(\begin{array}{cc}
\mathbf{0} & \mathbf{I} \\
\mathbf{H}_{f}^{1}(t) & \mathbf{H}_{f}^{2}(t)
\end{array}\right) \bar{Z}_{f}+\mathbf{G}_{f} \overline{W_{f}}(t)
$$

that generally could have a time-dependent form, when not only the frequency, but also the amplitude of loads, has an intrinsic evolutive nature. In $(8), \overline{W_{f}}(t)$ is a vector of $n_{f}$ white noise processes (stationary or nonstationary), $\mathbf{H}_{f}^{1}(t)$ and $\mathbf{H}_{f}^{2}(t)$ are two $m_{f} x m_{f}$ matrices, and $\mathbf{G}_{f}$ is a $2 m_{f} x n_{f}$ matrix that couples the excitation components of the forcing vector to the filter with a degree of freedom. Then, adopting the prefilter technique, the following system of motion differential equations is written in the state-space:

$$
\begin{gathered}
\left(\begin{array}{c}
\overline{\dot{X}}_{S} \\
\ddot{X}_{s}
\end{array}\right)=\left(\begin{array}{cc}
\mathbf{0} & \mathbf{I} \\
-\mathbf{M}^{-1} \mathbf{K} & -\mathbf{M}^{-1} \mathbf{C}
\end{array}\right)\left(\frac{\overline{X_{S}}}{\dot{X}_{S}}\right) \\
+\left(\begin{array}{cc}
\mathbf{0} & \mathbf{0} \\
\mathbf{G}_{s} \boldsymbol{\alpha}_{1}(t) & \mathbf{G}_{s} \boldsymbol{\alpha}_{2}(t)
\end{array}\right)\left(\begin{array}{l}
\bar{X}_{f} \\
\bar{X}_{f}
\end{array}\right), \\
\left(\begin{array}{cc}
\overline{\dot{X}}_{f} \\
\ddot{X}_{f}
\end{array}\right)=\left(\begin{array}{cc}
\mathbf{0} & \mathbf{I} \\
\mathbf{H}_{f}^{1}(t) & \mathbf{H}_{f}^{2}(t)
\end{array}\right)\left(\begin{array}{l}
\bar{X}_{f} \\
\dot{X}_{f}
\end{array}\right)+\mathbf{G}_{f} \bar{W}_{f}(t),
\end{gathered}
$$

which can be written also as

$$
\begin{aligned}
\left(\begin{array}{c}
\overline{\dot{X}}_{s} \\
\overline{\dot{X}}_{f} \\
\bar{X}_{s} \\
\overline{\ddot{X}}_{f}
\end{array}\right)= & \left(\begin{array}{cccc}
\mathbf{0} & \mathbf{0} & \mathbf{I} & \mathbf{0} \\
\mathbf{0} & \mathbf{0} & \mathbf{0} & \mathbf{I} \\
-\mathbf{M}^{-1} \mathbf{K} & \mathbf{G}_{s} \boldsymbol{\alpha}_{1}(t) & -\mathbf{M}^{-1} \mathbf{C} & \mathbf{G}_{s} \boldsymbol{\alpha}_{2}(t) \\
\mathbf{0} & \mathbf{H}_{f}^{1}(t) & \mathbf{0} & \mathbf{H}_{f}^{1}(t)
\end{array}\right) \\
& \times\left(\begin{array}{c}
\bar{X}_{s} \\
\bar{X}_{f} \\
\bar{X}_{S} \\
\bar{X}_{f}
\end{array}\right)+\left(\begin{array}{c}
\mathbf{0} \\
\mathbf{0} \\
\mathbf{0} \\
\mathbf{G}_{f}
\end{array}\right) \bar{W}_{f}
\end{aligned}
$$

and summarized as

$$
\overline{\dot{Z}}(t)=\mathbf{A}(t) \bar{Z}(t)+\mathbf{G}(t) \overline{W_{f}}(t),
$$

where

$$
\bar{Z}^{T}=\left(\bar{X}_{s}, \bar{X}_{f}, \bar{X}_{S}, \bar{X}_{f}\right)^{T}
$$

is a new global $2\left(m_{s}+m_{f}\right)$ state-space vector (structure plus filter).

In a design sensitivity problem, both the structural matrix and the response vector depend on a design parameter vector $\bar{b}$, whose elements may be structural stiffnesses, dampings, or masses, or any other mechanical parameters, as crosssections, Young modulus, boundary conditions, and so forth. Generally, also filter parameters and input intensity can be taken into account as design parameters. Thus, the matrix equation (11) must be explicitly re-written as:

$$
\overline{\dot{Z}}(b, t)=\mathbf{A}(\bar{b}, t) \bar{Z}(\bar{b}, t)+\mathbf{G} \overline{W_{f}}(t) .
$$

In case of zero initial condition, the solution of (13) has the general expression:

$$
\bar{Z}(\bar{b}, t)=\int_{0}^{t} \mathbf{\Phi}(\bar{b}, t, \tau) \mathbf{G} \overline{W_{f}}(\tau) d \tau,
$$

where the matrix $\Phi\left(\bar{b}, t_{1}, t_{2}\right)$ (e.g., see [1]) is usually called a transition matrix. The mean space-state vector $\bar{\mu}_{z}(t)$ can be determined by the differential vectorial equation:

$$
\overline{\dot{\mu}}_{z}(\bar{b}, t)=\mathbf{A}(\bar{b}, t) \bar{\mu}_{z}(\bar{b}, t)+\mathbf{G} \bar{\mu}_{w}(t),
$$

and the covariance matrix $\mathbf{R}_{Z Z}\left(\bar{b}, t_{1}, t_{2}\right)$ of the state-space $\bar{Z}(\bar{b}, t)$ is

$$
\mathbf{R}_{Z Z}\left(\bar{b}, t_{1}, t_{2}\right)=\left(\begin{array}{ll}
\mathbf{R}_{X X}\left(\bar{b}, t_{1}, t_{2}\right) & \mathbf{R}_{X \dot{X}}\left(\bar{b}, t_{1}, t_{2}\right) \\
\mathbf{R}_{\dot{X} X}\left(\bar{b}, t_{1}, t_{2}\right) & \mathbf{R}_{\dot{X} \dot{X}}\left(\bar{b}, t_{1}, t_{2}\right)
\end{array}\right) .
$$


This second-order symmetric statistical moments matrix $\mathbf{R}_{Z Z}\left(\bar{b}, t_{1}, t_{2}\right)$ has $\left(2 m^{2}+m\right)$ independent elements and can be evaluated by the well-known Lyapunov differential matrix equation

$$
\begin{aligned}
\dot{\mathbf{R}}_{Z Z}(\bar{b}, t)= & \mathbf{A}(\bar{b}, t) \mathbf{R}_{Z Z}(\bar{b}, t)+\mathbf{R}_{Z Z}(\bar{b}, t) \mathbf{A}(\bar{b}, t)^{T} \\
& +\mathbf{B}(\bar{b}, t)
\end{aligned}
$$

where

$$
\begin{aligned}
\mathbf{B}(\bar{b}, t) & =\left\langle\bar{Z}(\bar{b}, t) \mathbf{G}^{T} \bar{W}(t)^{T}\right\rangle+\left\langle\mathbf{G} \bar{W}(t) \bar{Z}^{T}(\bar{b}, t)\right\rangle \\
& =\mathbf{P}(\bar{b}, t)+\mathbf{P}^{T}(\bar{b}, t),
\end{aligned}
$$

and where $\mathbf{P}$ can be written as

$$
\begin{aligned}
\mathbf{P}(\bar{b}, t) & =\int_{0}^{t} \boldsymbol{\Phi}(\bar{b}, t, \tau) \mathbf{G}\left\langle\bar{W}(\tau) \bar{W}^{T}(t)\right\rangle \mathbf{G}^{T} d \tau \\
& =\int_{0}^{t} \boldsymbol{\Phi}(\bar{b}, t, \tau) \mathbf{G R}_{w w}(t, \tau) \mathbf{G}^{T} d \tau
\end{aligned}
$$

The matrix $\mathbf{B}(\bar{b}, t)$ can be simplified when the forcing vector is a white noise process as defined in (4) or in (7). In these cases, due to the properties of the Dirac function and of the transition matrix, the matrix $\mathbf{P}(t)$ is equal to

$$
\begin{aligned}
\mathbf{P}(t) & =\left(\int_{0}^{t} \boldsymbol{\Phi}(\bar{b}, t, \tau) \mathbf{G R}_{w w}(t, \tau) \mathbf{G}^{T} d \tau\right) \\
& =\boldsymbol{\Phi}(\bar{b}, 0) \mathbf{G R}_{w w}(t, t) \mathbf{G}^{T}=\mathbf{G} \mathbf{R}_{w w}(t, t) \mathbf{G}^{T}
\end{aligned}
$$

Finally, $\mathbf{B}(\bar{b}, t)$ can be written as

$$
\begin{aligned}
\mathbf{B}(t) & =\mathbf{G R}_{w w}(t, t) \mathbf{G}^{T}+\mathbf{G}^{T} \mathbf{R}_{w w}(t, t) \mathbf{G} \\
& =\left[\begin{array}{ll}
\mathbf{0}^{m x m} & \mathbf{0}^{m x m} \\
\mathbf{0}^{m x m} & \mathbf{L}(t)
\end{array}\right],
\end{aligned}
$$

where the $m_{f} x m_{f}$ submatrix $\mathbf{L}(t)$ is a diagonal matrix whose generic elements are

$$
[\mathbf{L}(t)]_{k, k}=\frac{2 \pi S_{0_{k}}}{m_{k}^{2}} \varphi_{k}^{2}(t) .
$$

For some applications, covariance information about structural acceleration is needed so that the matrix

$$
\mathbf{R}_{\ddot{X} \ddot{X}}(b, t)=\left\langle\ddot{\ddot{X}} \ddot{X}^{T}\right\rangle
$$

must be determined. This matrix is easily obtainable by the relation

$$
\mathbf{R}_{\bar{X} \bar{X}}(\bar{b}, t)=\mathbf{D}(\bar{b}, t) \mathbf{R}_{\bar{Z} \bar{Z}}(\bar{b}, t) \mathbf{D}(\bar{b}, t)^{T},
$$

where

$$
\begin{aligned}
& \mathbf{D}(\bar{b}, t) \\
& =\left[-\mathbf{M}(\bar{b})^{-1} \mathbf{K}(\bar{b}) \quad \mathbf{G}_{s} \boldsymbol{\alpha}_{1}(\bar{b}, t)-\mathbf{M}(\bar{b})^{-1} \mathbf{C}(\bar{b}) \quad \mathbf{G}_{s} \boldsymbol{\alpha}_{2}(\bar{b}, t)\right] .
\end{aligned}
$$

\section{Measure of Structural Reliability}

As stated previously, a wide class of structural dynamic problems deal with reliability evaluation. Due to the essential random nature of actions, a random dynamic analysis is necessary and the natural way of testing the structural integrity is to evaluate the probability whether a structure may not have a failure during its lifetime. Mechanical safety or reliability $r$ at a fixed time $T$ is then defined as a failure survival probability, where the failure is a partial or total static damage in a given time $[0, T]$. It is then clear that the definition of failure plays a central role in the evaluation of reliability, given the many possible interpretations. Normally, only two different kinds of mechanical failure are considered: fatigue failure, due to cumulative damage, and threshold crossing failure, determined by the first time crossing of a structural response parameter $s$ (able to describe failure conditions) through a given threshold value $\beta$. The present work deals with threshold crossing failure. Regarding a generic mechanical system subject to a stochastic process, the reliability $r(T)$ is defined as the survival probability for the exceeding of the given threshold value $\beta$ and, under the assumption that the initial survival probability is equal to one $(r(0)=1)$, is given by

$$
r(\bar{b}, T)=\exp \left\{-\int_{0}^{T} h(\beta, \bar{b}, t) d t\right\} .
$$

The hazard function $h(\beta, t)$ is defined as the probability of having a threshold crossing, in a unit time, in absence of previous threshold crossings. Its exact formulation is still an open question. In the Rice original formulation [15] for a single degree of freedom system, the hazard function has the following general form:

$$
h(\beta, \bar{b}, t)=\int_{0}^{+\infty} \dot{s} p_{S \dot{S}}(\beta, \bar{b}, \dot{s} \mid Q(t)) d \dot{s}
$$

in which $p_{S \dot{S}}(s, \dot{s} \mid Q(t))$ is the joint probability density of $S(t)$ and $\dot{S}(t)$ processes, and $Q(t)$ is the condition of absence of excursions of the fixed barrier $\beta$ before the time $t$. Difficulties related to the determination of this joint probability often impose the use of approximate solutions and one of which commonly used is the replacing of the conditional probability of failure with the unconditional probability $p_{S \dot{S}}(s, \dot{s})$. By means of this assumption, the hazard function $h(\beta, t)$ is replaced by the threshold crossing rate $v_{S}^{+}(\beta, t)$ which, under the further assumption that the above stochastic processes are Gaussian with null mean values, can be written as

$$
\begin{aligned}
v_{S}^{+}(\beta, \bar{b}, t)= & \frac{1}{2 \pi} a^{(1)}(\bar{b}, t) a^{(2)}(\bar{b}, t) \\
& \times a^{(3)}(\beta, \bar{b}, t) \chi\left[d_{S}(\beta, \bar{b}, t)\right],
\end{aligned}
$$




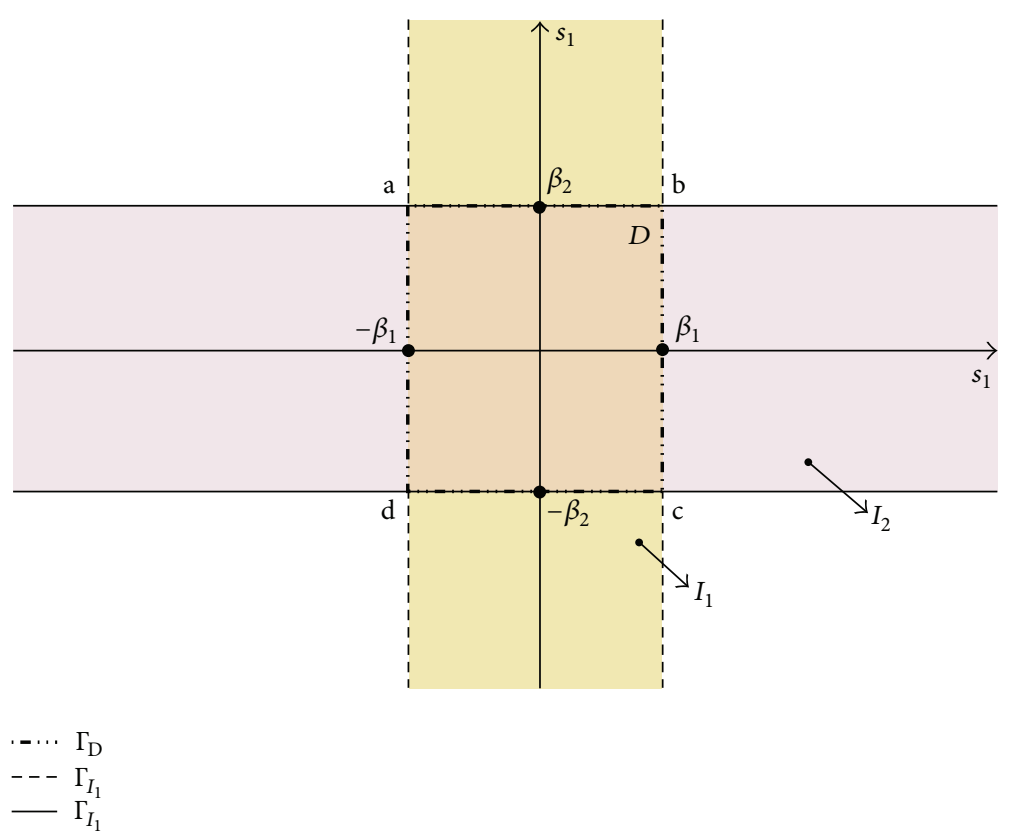

Figure 1: Plane safe domain $D$ (a-b-c-d) in a two-dimensional structural response parameters space $s_{1} \times s_{2}$.

where

$$
\begin{gathered}
a^{(1)}(\bar{b}, t)=\frac{\sigma_{\dot{S}}(\bar{b}, t)}{\sigma_{S}(\bar{b}, t)}, \\
a^{(2)}(\bar{b}, t)=\sqrt{1-\rho_{S \dot{S}}^{2}(\bar{b}, t)} \\
a^{(3)}(\beta, \bar{b}, t)=\exp \left\{-\frac{1}{2}\left(\frac{\beta}{\sigma_{S}(\bar{b}, t)}\right)^{2}\right\}, \\
\left.\chi d_{S}(\beta, \bar{b}, t)\right]=\exp \left(-\frac{d_{S}(\beta, \bar{b}, t)^{2}}{2}\right) \\
+d_{S}(\bar{b}, t) \sqrt{2 \pi}\left[1-\Phi\left(d_{x}(\beta, \bar{b}, t)\right)\right] \\
d_{S}(\bar{b}, t)=\frac{\beta}{\sigma_{S}(\bar{b}, t)}\left(\frac{\rho_{S \dot{S}}(\bar{b}, t)}{\sqrt{1-\rho_{S \dot{S}}^{2}(\bar{b}, t)}}\right) .
\end{gathered}
$$

In $(30), \sigma_{S}(\bar{b}, t)$ and $\sigma_{\dot{S}}(\bar{b}, t)$ are the standard deviations of $S(\bar{b}, t)$ and $\dot{S}(\bar{b}, t)$, and $\rho_{S \dot{S}}(\bar{b}, t)$ is their correlation factor. For a structural system, the threshold crossing rate $v_{s}^{+}$of a global safety domain $D \subset R^{m}$ can be determined by means of the following extension of the Rice formula [16], regarding a generic multidimensional stochastic process, and here referred to as $n_{s^{x}} 1$ internal force vector $\mathbf{s}$, whose elements are suitable structural response parameters (internal forces or stresses evaluated in a finite number of critical sections or points):

$$
v_{\mathbf{s}}^{+}=\int_{\Gamma_{D}}\left[\int_{\overline{\dot{s}} \cdot \bar{n} \geq 0}(\overline{\dot{s}} \cdot \bar{n}) p_{\mathbf{S s}}(\bar{s}, \overline{\dot{s}}, \bar{b}) d \overline{\dot{s}}\right] d \bar{s},
$$

where $\Gamma_{D}$ is a safety domain boundary and $\bar{n}$ is the outer normal to the boundary.

Though an exact evaluation of reliability based on (31) is conceptually possible, it presents some difficulties. Many different simplified approaches have been proposed in last twenty years, when outcrossing rates of a vector random process have been studied in the context of problems in load combinations and in structural reliability [17-23].

In this work, an approximate and conservative evaluation of $v_{\mathrm{s}}^{+}$is preferred as it can be easily adopted when the safe domain $D$ is a hyper rectangle in $R^{m}$ (Figure 1 )

$$
D=\left[-\beta_{1}, \beta_{1}\right] \times\left[-\beta_{2}, \beta_{2}\right] \times \cdots \times\left[-\beta_{m}, \beta_{m}\right],
$$

and $v_{\mathrm{s}}^{+}$is evaluated as the sum of the mean crossing rate on each face of the hyper rectangle $D$. In this simplified approach, it is assumed that $v_{s}^{+}$is the sum of the crossing rates through each hyper plane on which the faces of $D$ lie. For instance, in the two-dimensional space $R^{2}$, the safe domain $D$ is the rectangle $I_{1} \cap I_{2}$, as shown in Figure 1, and (32) is an integral evaluated on the rectangular boundary $\Gamma_{D}$. The safe domain boundary $\Gamma_{D}$ is replaced by $\Gamma_{I_{1}} \cup \Gamma_{I_{2}}$, and $v_{s}^{+}$is evaluated as the sum of the crossing rates $v_{1}^{+}$and $v_{2}^{+}$out of the regions $I_{1}$ and $I_{2}$, respectively. 


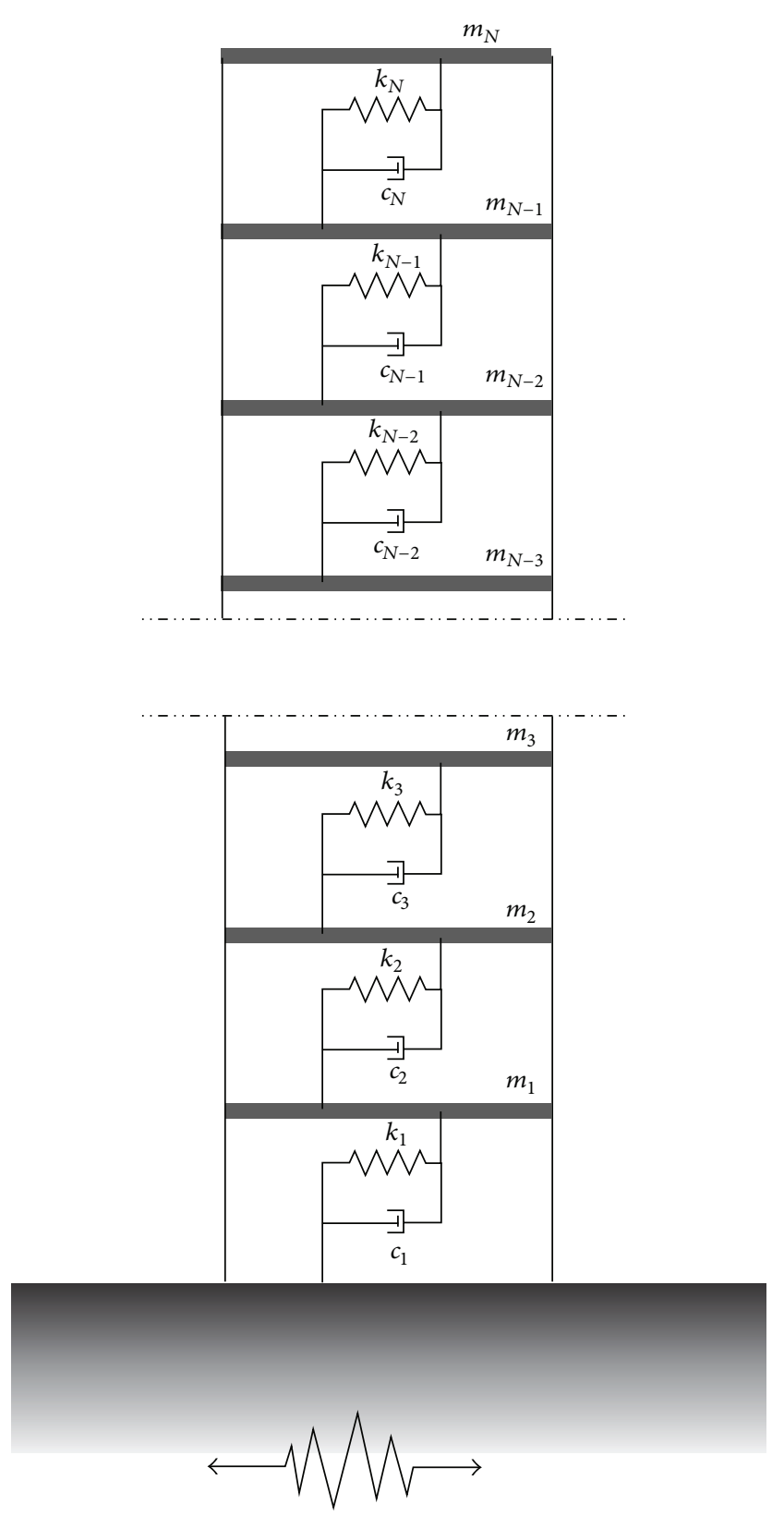

FIGURE 2: Mechanical scheme of analysed plane shear type.

Generally, the safe domain can be obtained as

$$
D=I_{1} \cap \cdots \cap I_{i} \cdots \cap I_{m}
$$

where $I_{i}=[-\infty, \infty] \times \cdots \times\left[-\beta_{i}, \beta_{i}\right] \times \cdots \times[-\infty, \infty]$. Since the integrand function in (31) is always nonnegative and given

$$
\Gamma_{D} \subset\left(\bigcup_{i=1}^{n} \Gamma_{I_{1}}\right),
$$

we have that

$$
v_{\mathrm{s}}^{+} \leq \sum v_{i}^{+}
$$

where

$$
\begin{aligned}
v_{i}^{+}= & \int_{-\infty}^{0} \dot{s} p_{S_{i} \dot{s}_{i}}\left(-\beta_{i}, \bar{b}, \dot{s}_{i}\right) d \dot{s}_{i} \\
& +\int_{0}^{+\infty} \dot{s} p_{S_{i} \dot{S}_{i}}\left(\beta_{i}, \bar{b}, \dot{s}_{i}\right) d \dot{s}_{i}
\end{aligned}
$$

and in this particular case with symmetric barriers, we have

$$
v_{i}^{+}=2 \int_{0}^{+\infty} \dot{s} p_{S_{i} \dot{S}_{i}}\left(\beta_{i}, \bar{b}, \dot{s}_{i}\right) d \dot{s}_{i} .
$$

In the following, $v_{\mathrm{s}}^{+}$will always be evaluated as $\sum v_{i}^{+}$. It must be noted that (35) is strictly satisfied in case of rare events.

The final structural reliability is evaluated as the product of all reliability evaluated separately for each structtural response parameter. Using this approach, it is possible to define a reliability vector $\bar{r}(t)$ as the collection of independent reliability $r_{h}(t)$ related to the first time crossing of a structural response parameter $s_{h}$ through given symmetric threshold values $\pm \beta_{h}$ that induce a structural failure.

It is then necessarily required to evaluate the probability $P_{f, h}$ that the absolute value $\left|s_{h}\right|$ exceeds $\beta_{h}$, at least once during all input duration. The generic reliability vector element $r_{h}(T)$ is defined as

$$
r_{h}(\bar{b}, T)=P\left[S_{h} \leq \beta_{h} ; t \in[0, T]\right],
$$

where, under the Poisson hypothesis, we have:

$$
\begin{aligned}
& r_{h}(\bar{b}, T) \\
&=\exp \left\{-\frac{1}{\pi} \int_{0}^{T}\left(\frac{\sigma_{\dot{S}_{h}}(\bar{b}, t) \sqrt{1-\rho_{S_{h} \dot{S}_{h}}^{2}(\bar{b}, t)}}{\sigma_{S_{h}}(\bar{b}, t)}\right.\right. \\
& \times \exp \left\{-\frac{1}{2}\left(\frac{\beta_{h}}{\sigma_{S_{h}}(\bar{b}, t)}\right)^{2}\right\} \\
&\left.\left.\times \chi\left[d_{S_{h}}(\bar{b}, t)\right]\right) d \tau\right\},
\end{aligned}
$$

where $\sigma_{S_{h}}^{2}, \sigma_{\dot{S}_{h}}^{2}$, and $\rho_{S \dot{S}_{h}}$ are, respectively, the $h$ and the $n+h$ diagonal elements of $\mathbf{R}_{Z_{S} Z_{S}}(t)$, where $\overline{Z_{S}}=(\bar{s} \dot{S})^{T}$ and $\eta_{S_{h}}=$ $\beta_{h} / \sigma_{S_{h}}$.

With reference to a vector of response functions of interest $\bar{S}(t)$ in reliability evaluation, related to the state space vector $\bar{Z}(t)$ by the linear transformation $\bar{S}(t)=\mathbf{T}_{1}(\bar{b}) \bar{X}(t)$, $\overline{\dot{S}}(t)=\mathbf{T}_{2}(\bar{b}) \bar{Z}(t)$, the general form to express it become

$$
\bar{Y}(t)=(\bar{S} \quad \overline{\dot{S}})^{T}=\overline{\mathbf{T}}(\bar{b}) \bar{Z}(t),
$$

where

$$
\overline{\mathbf{T}}(\bar{b})=\left(\begin{array}{cc}
\mathbf{T}_{1}(\bar{b}) & \mathbf{0} \\
\mathbf{0} & \mathbf{T}_{2}(\bar{b})
\end{array}\right),
$$


and the covariance matrix in structural response parameters space-state $\bar{Z}_{S}$ is

$$
\mathbf{R}_{Z_{S} Z_{S}}(\bar{b}, t)=\overline{\mathbf{T}}(\bar{b}) \mathbf{R}_{Z Z}(\bar{b}, t) \hat{\mathbf{T}}^{T}(\bar{b})
$$

The final global structural reliability is then

$$
r_{\text {global }}(\bar{b}, t)=\prod_{h=1}^{n} r_{h}(\bar{b}, t) \text {. }
$$

The equation represents the probability that the $h$ th interfloor displacement will cross the maximum acceptable value $\beta_{h}$ during time interval $[0, T]$. All the barriers can be collected in the barrier vector $\bar{\beta}$.

In case of failure due to a double symmetric threshold crossing, the threshold crossing rate $v_{i}^{+}$in the generic $i$ th critical node is given by

$$
v_{i}(\bar{b}, t)=2 v_{i}^{+}\left(\bar{b}, \beta_{i}, t\right)
$$

where $v_{i}^{+}\left(\beta_{i}, t\right)$ is determined by (37).

$$
\mathbf{A}_{, b_{j}}(\bar{b}, t)=\left(\begin{array}{cccc}
\mathbf{0} & \mathbf{0} & \mathbf{I} & \mathbf{0} \\
\mathbf{0} & \mathbf{0} & \mathbf{0} & \mathbf{I} \\
\left(-\mathbf{M}(\bar{b})^{-1} \mathbf{K}(\bar{b})\right)_{, b_{j}} & \mathbf{G}_{s} \boldsymbol{\alpha}_{1}(t) & \left(-\mathbf{M}(\bar{b})^{-1} \mathbf{C}(\bar{b})\right)_{, b_{j}} & \mathbf{G}_{s} \boldsymbol{\alpha}_{2}(t) \\
\mathbf{0} & \mathbf{H}_{f}^{1}(t) & \mathbf{0} & \mathbf{H}_{f}^{1}(t)
\end{array}\right)
$$

in which it has been assumed that input parameters do not depend on the design vector $\bar{b}$. In (47), we have

$$
\begin{gathered}
\left(-\mathbf{M}^{-1} \mathbf{K}\right)_{, b_{j}}=\mathbf{M}^{-1} \mathbf{M}_{, b_{j}} \mathbf{M}^{-1} \mathbf{K}-\mathbf{M}^{-1} \mathbf{K}_{, b_{j}}, \\
\left(-\mathbf{M}^{-1} \mathbf{C}\right)_{, b_{j}}=\mathbf{M}^{-1} \mathbf{M}_{, b_{j}} \mathbf{M}^{-1} \mathbf{C}-\mathbf{M}^{-1} \mathbf{C}_{b_{j}} .
\end{gathered}
$$

To determine reliability sensitivity in structural response parameter space $\bar{S}$, it is necessary to obtain

$$
\begin{aligned}
\left\{\mathbf{R}_{Z_{s} Z_{S}}(\bar{b}, t)\right\}_{, b_{j}}= & \{\hat{\mathbf{T}}(\bar{b})\}_{, b_{j}} \mathbf{R}_{Z Z}(\bar{b}, t) \hat{\mathbf{T}}^{T}(\bar{b}) \\
& +\overline{\mathbf{T}}(b)\left\{\mathbf{R}_{Z Z}(\bar{b}, t)\right\}_{, b_{j}} \hat{\mathbf{T}}^{T}(\bar{b}) \\
& +\overline{\mathbf{T}}(\bar{b}) \mathbf{R}_{Z Z}(\bar{b}, t)\left\{\hat{\mathbf{T}}^{T}(\bar{b})\right\}_{, b_{j}},
\end{aligned}
$$

and thus the generic $i$ th reliability sensitivity is

$$
\begin{aligned}
\left\{r_{S_{i}}(\beta, \bar{b}, t)\right\}_{, b_{j}}= & \left(\int_{0}^{T}\left\{v_{S_{i}}(\beta, \bar{b}, \tau)\right\}_{, b_{j}} d \tau\right) \\
& \times \exp \left[\int_{0}^{T} v_{S_{i}}(\beta, \bar{b}, \tau) d \tau\right],
\end{aligned}
$$

3.1. Response Reliability Sensitivity. After covariance matrix $\mathbf{R}_{z z}$ has been determinated, its first order sensitivity, whose determination is necessary in determining the structural reliability sensitivity, is evaluated (in the following, the notations ${ }_{, b_{j}}=\{\cdot\}_{, b_{j}}=\partial \cdot / \partial b_{j}$ will be adopted). Consider

$$
\mathbf{R}_{Z Z, b_{j}}=\frac{\partial \mathbf{R}_{Z Z}(\bar{b}, t)}{\partial b_{j}}
$$

In case of nonstationary conditions, the design sensitivity $\mathbf{R}_{Z Z, b_{j}}$ of the covariance matrix $\mathbf{R}_{Z Z}$ is determined by solving the following matrix differential equation:

$$
\begin{aligned}
\frac{\dot{\mathbf{R}}(\bar{b}, t)}{\partial b_{j}}= & \dot{\mathbf{R}}_{b_{j}}(t) \\
= & \mathbf{A}(\bar{b}, t) \mathbf{R}_{b_{j}}(\bar{b}, t)+\mathbf{R}_{b_{j}}(\bar{b}, t) \mathbf{A}(\bar{b}, t)^{T} \\
& +\left(\mathbf{A}_{b_{j}} \mathbf{R}+\mathbf{R A}_{, b_{j}}^{T}\right) .
\end{aligned}
$$

The $2\left(m_{s}+m_{f}\right) \times 2\left(m_{s}+m_{f}\right)$ matrix $\mathbf{A}_{, b_{i}}$ has the following expressions which, without loss of generalities, can been simplified as: where the mean rate crossing failure sensitivity is (see details in Appendix B)

$$
\begin{aligned}
\left\{v_{S_{i}}^{+}(\beta, \bar{b}, t)\right\}_{, b_{j}}=\frac{1}{\pi}( & \left\{a^{1}\right\}_{, b_{j}} a^{2} a^{3} \chi+a^{1}\left\{a^{2}\right\}_{, b_{j}} a^{3} \chi \\
& \left.+a^{1 a^{2}}\left\{a^{3}\right\}_{, b_{j}} \chi+a^{1} a^{2} a^{3}\{\chi\}_{, b_{j}}\right) .
\end{aligned}
$$

In view of (42), the first order sensitivity of the global reliability is then

$$
\left\{r_{\text {global }}(\bar{b}, t)\right\}_{, b_{j}}=\sum_{i=1}^{n_{s}}\left(\left\{r_{i}(\bar{b}, t)\right\}_{, b_{j}} \prod_{k=1, k \neq i}^{n_{s}} r_{k}(\bar{b}, t)\right) .
$$

\section{Numerical Example}

In the present application of the proposed method, a multistorey building subject to an earthquake excitation is taken into consideration. For simplicity, and without loss of the generalities in the proposed technique, the building model adopted is a generic shear type plane frame structure, as shown in Figure 2. When floor slabs in a building generally have a very large degree of stiffness in-plane, then they can be regarded as rigid diaphragms. This increases analysis 
efficiency greatly without any significant loss of accuracy in the assessment of the response resulting from ground excitations. In addition to the rigid floor diaphragm assumption, the efficiency of computation can be further increased by applying the matrix condensation technique.

The main building mechanical model is assumed to be linear. This is an acceptable hypothesis within the horizontal displacement limitations required to guarantee an operative service level. In other words, this simplified hypothesis is perfectly functional when the assigned maximum interstorey drift is quite close to the elastic limit of the structural displacement (e.g., in case of full operatives demand). The design vector is assumed to be in order composed by each floor mass, stiffness, and damping:

$$
\bar{b}=\left(\bar{b}_{1}, \bar{b}_{2}, \bar{b}_{3}\right)=\left(\bar{m}^{T}, \bar{k}^{T}, \bar{c}^{T}\right),
$$

where

$$
\begin{gathered}
\bar{b}_{1}=\bar{m}^{T}=\left(m_{1}, m_{2}, m_{3}, \ldots, m_{m_{s}}\right), \\
\bar{b}_{2}=\bar{k}^{T}=\left(k_{1}, k_{2}, k_{3}, \ldots, k_{m_{s}}\right), \\
\bar{b}_{3}=\bar{c}^{T}=\left(c_{1}, c_{2}, c_{3}, \ldots, c_{m_{s}}\right) .
\end{gathered}
$$

4.1. Motion Equation. To preserve the main seismic peculiarity of spectral and time modulation, a nonstationary modulated Kanai-Tajimi process is used for stochastic ground motion. It describes the base acceleration $\ddot{X}_{g}(t)$ applied at the base of the structure as

$$
\begin{gathered}
\ddot{X}_{g}(t)=\ddot{X}_{f}(t)+\varphi(t) w(t), \\
\ddot{X}_{f}(t)+2 \xi_{f} \omega_{f} \dot{X}_{f}(t)+\omega_{f}^{2} X_{f}(t)=-\varphi(t) w(t),
\end{gathered}
$$

where $X_{f}(t)$ is the response of the Kanai-Tajimi filter, having frequency $\omega_{f}$ and damping coefficient $\xi_{f}$, and $w(t)$ is the white noise whose constant bilateral Power Density Spectral (PDS) function is $S_{0}$. This last parameter is related to the Peak Ground Acceleration (PGA) $\ddot{X}_{g}^{\max }$ by means of relation [24]

$$
S_{0}=0.2222 \frac{\xi_{g}\left(\ddot{X}_{g}^{\max }\right)^{2}}{\pi \omega_{g}\left(1+4 \xi_{g}^{2}\right)} .
$$

The nonstationary character of the problem is introduced by the deterministic temporal modulation function $\varphi(t)$, which controls the intensity variation, without changing earthquake frequency contents. In this case, the modulation function proposed by Jennings [25] is adopted as follows:

$$
\varphi(t)= \begin{cases}\left(\frac{t}{t_{1}}\right)^{2} & t<t_{1} \\ 1 & t_{1} \leq t \leq t_{2} \\ e^{-\beta\left(t-t_{2}\right)} & t>t_{2} .\end{cases}
$$

The system of motion equations for the complete structural system then are

$$
\begin{aligned}
& \ddot{X}_{s}(t)+\mathbf{M}(\bar{b})^{-1} \mathbf{C}(\bar{b}) \overline{\dot{X}}_{s}(t)+\mathbf{M}(\bar{b})^{-1} \mathbf{K}(\bar{b}) \bar{X}_{s}(t) \\
&=-\bar{r}\left(2 \xi_{f} \omega_{f} \dot{X}_{f}(t)+\omega_{f}^{2} X_{f}(t)\right), \\
& \ddot{X}_{f}(t)+2 \xi_{f} \omega_{f} \dot{X}_{f}(t)+\omega_{f}^{2} X_{f}(t)=-w(t) \varphi(t) .
\end{aligned}
$$

It can be written in matrix form as

$$
\begin{aligned}
\left(\begin{array}{c}
\overline{\dot{X}}_{s} \\
\overline{\dot{X}}_{f} \\
\overline{\ddot{X}}_{s} \\
\overline{\ddot{X}}_{f}
\end{array}\right)= & \left(\begin{array}{cccc}
\mathbf{0} & \mathbf{0} & \mathbf{I} & \mathbf{0} \\
0 & 0 & 0 & 1 \\
-\mathbf{M}^{-1} \mathbf{K} & -\omega_{f}^{2} \bar{r} & -\mathbf{M}^{-1} \mathbf{C} & -2 \xi_{f} \omega_{f} \bar{r} \\
0 & -\omega_{f}^{2} & 0 & -2 \xi_{f} \omega_{f}
\end{array}\right. \\
& \times\left(\begin{array}{c}
\bar{X}_{s} \\
\bar{X}_{f} \\
\bar{X}_{S} \\
\overline{\dot{X}}_{f}
\end{array}\right)+\left(\begin{array}{c}
\overline{0} \\
\overline{0} \\
\overline{0} \\
-\varphi(t)
\end{array}\right) w(t),
\end{aligned}
$$

where $\bar{r}=[1,1,1, \ldots, 1,1]^{T}$ is the $m_{s} x 1$ drag vector, and $\mathbf{M}(\bar{b})$, $\mathbf{C}(\bar{b})$, and $\mathbf{K}(\bar{b})$ are, respectively, the mass, viscosity, and stiffness $m_{s} x m_{s}$ structural matrices, whose general expression is

$$
\begin{aligned}
& \mathbf{K}=\left(\begin{array}{ccc}
k_{1}+k_{2} & -k_{2} & 0 \\
-k_{2} & k_{2}+k_{3} & -k_{3} \\
0 & -k_{3} & k_{3}
\end{array}\right) \\
& \mathbf{C}=\left(\begin{array}{ccc}
c_{1}+c_{2} & -c_{2} & 0 \\
-c_{2} & c_{2}+c_{3} & -c_{3} \\
0 & -c_{3} & c_{3}
\end{array}\right) \\
& \mathbf{M}=\operatorname{diag}\left(m_{1}, m_{2}, m_{3}\right) .
\end{aligned}
$$

The three vectors $\bar{X}_{s}(\bar{b}, t), \overline{\dot{X}}_{s}(\bar{b}, t)$, and $\bar{X}_{s}(\bar{b}, t)$ are acceleration, velocity, and displacement $n_{x} 1$ vectors, relative to the ground. In the analyzed structure, the mass matrix is diagonal and the two viscous and stiffens matrices are tridiagonal. The matrix differential equation (59) is also written as

$$
\overline{\dot{Z}}(\bar{b}, t)=\mathbf{A}(\bar{b}) \bar{Z}(\bar{b}, t)+\bar{F}(t) .
$$

4.2. Reliability Evaluation. With reference to the proposed reliability problem, the probability $P_{f, h}$ that each $h$ th storey drift $U_{h}$ exceeds the threshold $\beta_{h}$ at least once in a given interval must be evaluated. Then, for each $h$ th floor, this failure event is associated with the condition that $\left|x_{h+1}-x_{h}\right|=$ $\left|u_{h}\right|=\beta_{h}$. The corresponding reliability vector element is

$$
r_{h}\left(\bar{b}, \beta_{h}, T\right)=P\left[\left|U_{h}(\bar{b}, t)\right| \leq \beta_{h} ; t \in[0, T]\right] .
$$




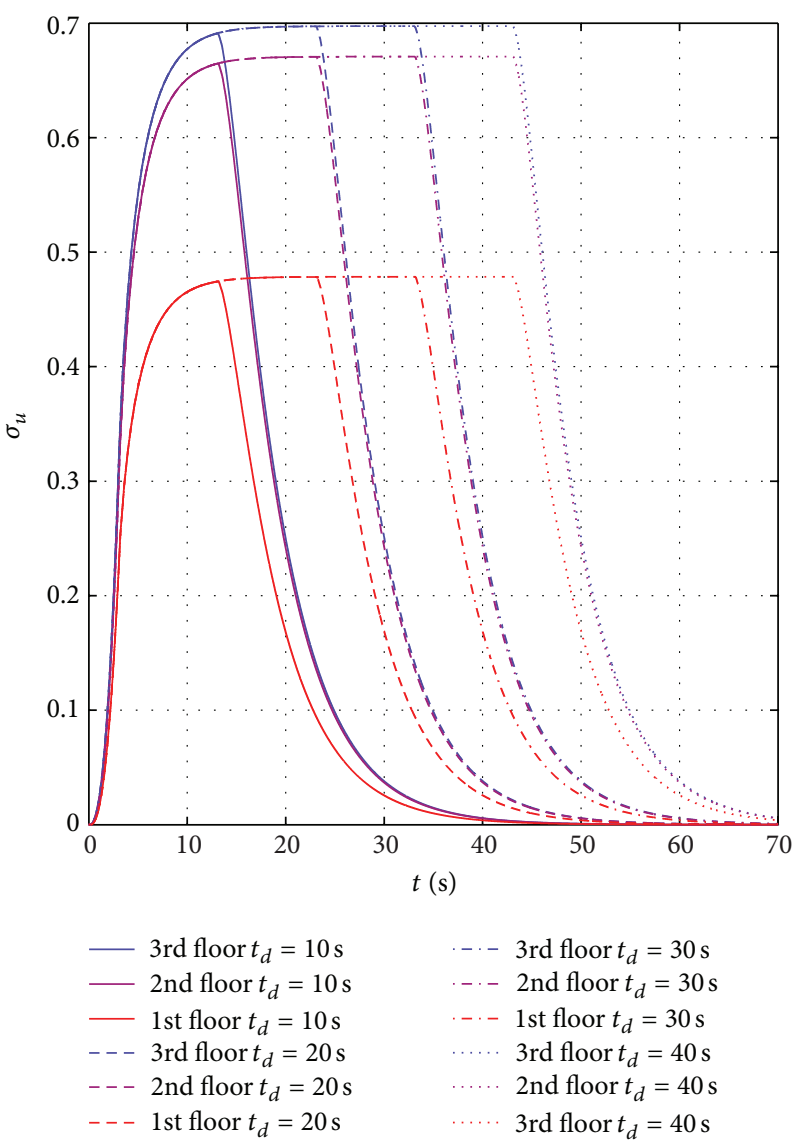

(a)

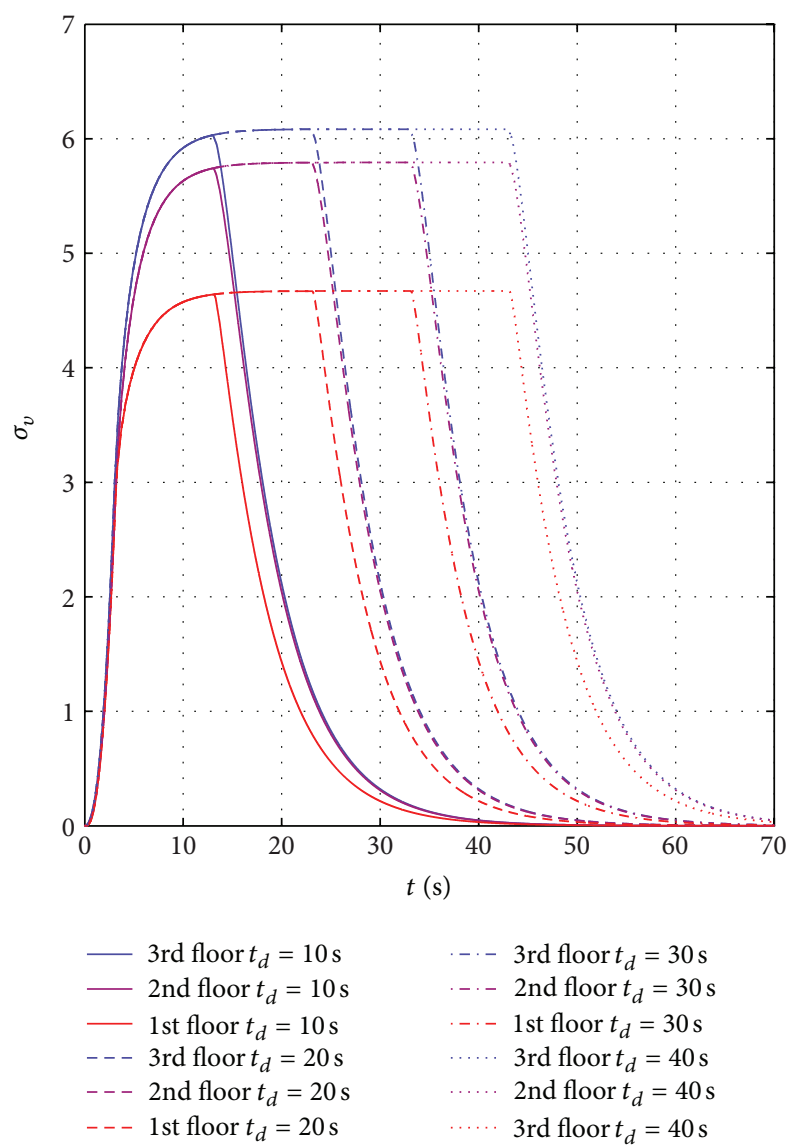

(b)

FiguRE 3: Interstorey displacement $\left((\mathrm{a}), \sigma_{u}\right)$ and velocities $\left((\mathrm{b}), \sigma_{v}\right)$ response of a 3-DoF (different colors for different levels) system subject to modulated filtered white noise with dissimilar time durations (different line-types).

Thus, assuming the Poissonian crossing hypothesis

$$
\begin{aligned}
r_{h}\left(\bar{b}, \beta_{h}, T\right) & \\
=\exp \left\{-\frac{1}{\pi} \int_{0}^{T}\right. & \left(\frac{\sigma_{\dot{U}_{h}}(\bar{b}, \tau)}{\sigma_{U_{h}}(\bar{b}, \tau)} \sqrt{1-\rho_{U_{h} \dot{U}_{h}}^{2}(\bar{b}, t)}\right. \\
& \times \exp \left\{-\frac{1}{2} \eta_{h}^{2}\left(\bar{b}, \beta_{h}, \tau\right)\right\} \\
& \left.\left.\times \chi\left[d_{U_{h}}\left(\bar{b}, \beta_{h}, t\right)\right]\right) d \tau\right\},
\end{aligned}
$$

and the final global structural reliability is then determined as:

$$
r_{\text {global }}(\bar{b}, \bar{\beta}, t)=\prod_{h=1}^{n} r_{h}\left(\bar{b}, \beta_{h}, t\right) .
$$

Although exact analytical solutions for $r_{\text {global }}(\bar{b}, \bar{\beta}, t)$ are generally definable from the theoretical point of view, it is known that (64) is an approximate upper-bound of global reliability and provides a conservative estimation of $r_{\text {global }}(t)$, as previous stated. Moreover, it can be efficiently used for design and predesign purposes in practical engineering problems.

In order to evaluate the reliability vector related to the interfloor relative displacement threshold crossing, the interstorey drift vector is introduced as

$$
\bar{u}(t)=\left[x_{1}, x_{2}-x_{1}, x_{3}-x_{2}, \ldots, x_{n}-x_{n-1}, x_{f}\right]^{T}
$$

whose covariance matrix $R_{Z_{U} Z_{U}}(t)$ must be evaluated.

The elements of the linear space of stochastic processes are then $\bar{Z}_{U}(\bar{b})=\left[\bar{U}^{T}(\bar{b}), \overline{\dot{U}}^{T}(\bar{b})\right]^{T}$. The linear equation $U(\bar{b}, t)=\mathbf{T} \bar{X}(\bar{b}, t)$ relates the interstorey drift vector $\bar{U}(\bar{b}, t)$ 


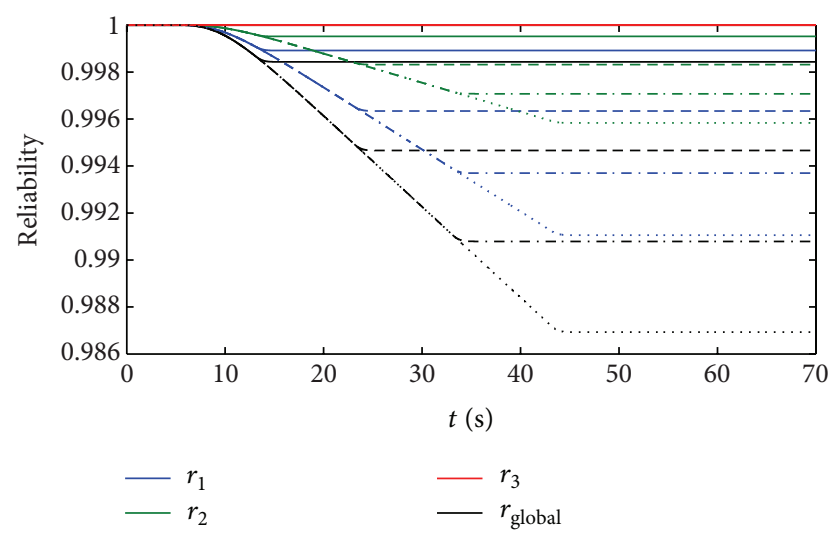

FIGURE 4: Structural reliability of a 3-DoF system, evaluated as the probability of maximum interstorey drift exceeds over a given threshold of $3 \mathrm{~cm}$.

and the storey displacement vector $\bar{X}(\bar{b}, t)$, where the transform matrix $\mathbf{T}$ is bidiagonal and is independent from design vector

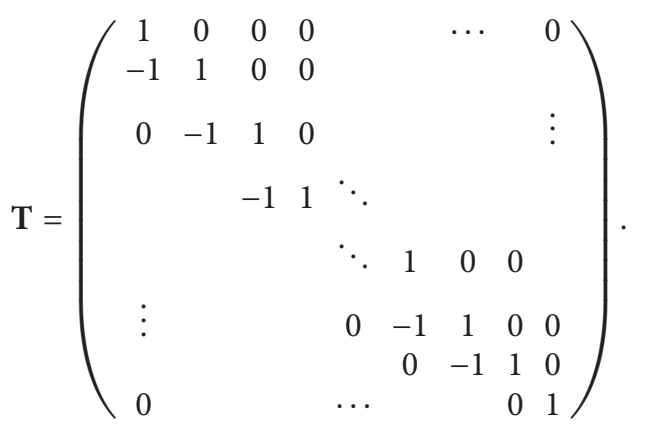

The covariance matrix $\mathbf{R}_{Z_{U} Z_{U}}(\bar{b}, t)$ is then related to $\mathbf{R}_{Z Z}(\bar{b}, t)$ through the following relations:

$$
\begin{gathered}
\mathbf{R}_{Z_{U} Z_{U}}(\bar{b}, t)=\widehat{\mathbf{T}} \mathbf{R}_{Z Z}(\bar{b}, t) \widehat{\mathbf{T}}^{T}, \\
\widehat{\mathbf{T}}=\left(\begin{array}{ll}
\mathbf{T} & \mathbf{0} \\
\mathbf{0} & \mathbf{T}
\end{array}\right) .
\end{gathered}
$$

The reliability vector $\bar{r}_{U}$ previously defined can be evaluated as the collection of

$$
r_{U h}(T)=r_{0} e^{-\int_{0}^{T} v_{U h}^{+}(\tau) d \tau},
$$

where $v_{U_{h}}^{+}\left(\eta_{V_{h}}\right)$ is function of $\sigma_{U_{h}}^{2}, \sigma_{\dot{U}_{h}}^{2}, \rho_{U_{h} \dot{U}_{h}}$, and $\eta_{U_{h}}=$ $\beta_{h} / \sigma_{U_{h}}$. The first two are, respectively, the $h$ and the $n+h$ main diagonal elements of $\mathbf{R}_{Z_{U} Z_{U}}(t)$, the third is the $h, n+$ $h$ element of the same matrix, and finally $\beta_{h}$ is the $h$ th barrier. Equation (68) represents the probability that the $h$ th interfloor displacement will cross the maximum acceptable value $\beta_{h}$ during time interval $[0, T]$. All the barriers are collected in the vector $\bar{\beta}$. In the example here assumed its elements are all equal to $3.0 \mathrm{~cm}$ for each floor, which corresponds to a lateral drift of $1.0 \%$ in case of $3 \mathrm{~m}$ interstorey.
4.3. System Parameters. In the building configurations here adopted, there are three equal mass storeys $m_{i}=1.50 \times$ $10^{5}(\mathrm{~kg})$. First floor lateral stiffness is $k_{1}=6.0 \cdot 10^{7}(\mathrm{~N} / \mathrm{m})$, and it is assumed that a linear stiffness reduction of $30 \%$ takes place at the upper floors, so that $k_{2}=5.1 \cdot 10^{7}(\mathrm{~N} / \mathrm{m})$ and $k_{3}=4.2 \cdot 10^{7}(\mathrm{~N} / \mathrm{m})$. Finally, damping is evaluated by setting $c_{i}=2 \sqrt{m_{i} k_{i}}$, so that $c_{1}=3 \times 10^{5} \mathrm{Nsec} / \mathrm{m}, c_{2}=$ $2,8 \times 10^{5} \mathrm{Nsec} / \mathrm{m}, c_{3}=2.5 \times 10^{5} \mathrm{Nsec} / \mathrm{m}$. The seismic characterization assumes a peak ground acceleration (PGA) of $0.45(\mathrm{~g})$, and 4 different total earthquake durations $t_{d}=$ $t_{2}-t_{1}$ are evaluated. They are, respectively, equal to 10, 20, 30 and 40 seconds.

In Figure 3 the structural interstorey covariances are shown, in terms of displacement (a) and velocity (b). Continuous lines are for $t_{d}=10 \mathrm{sec}$., dashed lines are for $t_{d}=20$ sec., dash-dot lines are for $t_{d}=30 \mathrm{sec}$., and finally dotted lines are for $t_{d}=40 \mathrm{sec}$. Blue lines are for first level covariance, magenta lines are for second level, and red lines are for the $3^{\text {dh }}$ level. It must be noticed that structural responses reach a stationary level if $t_{d}$ is longer than $10 \mathrm{sec}$. Moreover, for this specific structural configuration, covariance responses of the first level are the greatest; the second is only slightly smaller than the first and the third is about the half.

In Figure 4, reliabilities are shown and structural safeties are evaluated for each lateral interstorey drift threshold crossing, and global reliability is evaluated as approximate upper-bound global reliability. Also in this figure, line type and line colours are used as in Figure 3. Moreover, here black lines are for global system reliability. It should be noticed that the first interstorey drift failure is the more probable, the second one has just a smaller failure probability, and finally the third one has a quite negligible failure probability (i.e., $r_{3}=1$ ).

In Figure 5, first order sensitivities of lateral interstorey drifts evaluated by the proposed approach are shown, for different time durations and storey levels. Results are plotted by using a nondimensional measure, represented by the parameter

$$
\left(\sigma_{u_{i}}(t)\right)_{, b_{j}}^{\mathrm{AD}}=\frac{\partial \sigma_{u_{i}}(t)}{\partial b_{j}} \frac{b_{j}}{\left(\sigma_{u_{i}}\right)_{\mathrm{MAX}}} .
$$

Sensitivities are evaluated with regards to three sets of mechanical parameters: the three floor masses (in graphs a, $\mathrm{d}$, and $\mathrm{g}$ ), the three floor stiffness (in graphs b, e, and $\mathrm{h}$ ), and finally the three floor dampings (in graphs $c, f$, and i).

With reference to the first set of mechanical parameters, it must be noticed that the increase of floor masses (blue for the first one, magenta for the second, and red for the third one) has the effect of increasing lateral drift covariance responses. This effect takes place in any analysed case. Moreover, it must be also noticed that the first interstorey drift sensitivity has about the same values with respect to each three structural masses, as it is extremely evident in Figure 5(a). On the contrary, the second interstorey drift Figure 5(b) is more sensible to $m_{2}$ (magenta) and $m_{3}$ (red), then to $m_{1}$ (blue). Finally, the third interstorey drift Figure 5(c) is more sensible with respect to $m_{3}$ than to the other two masses. This is due to the fact that in general, a change of masses in a position lower 


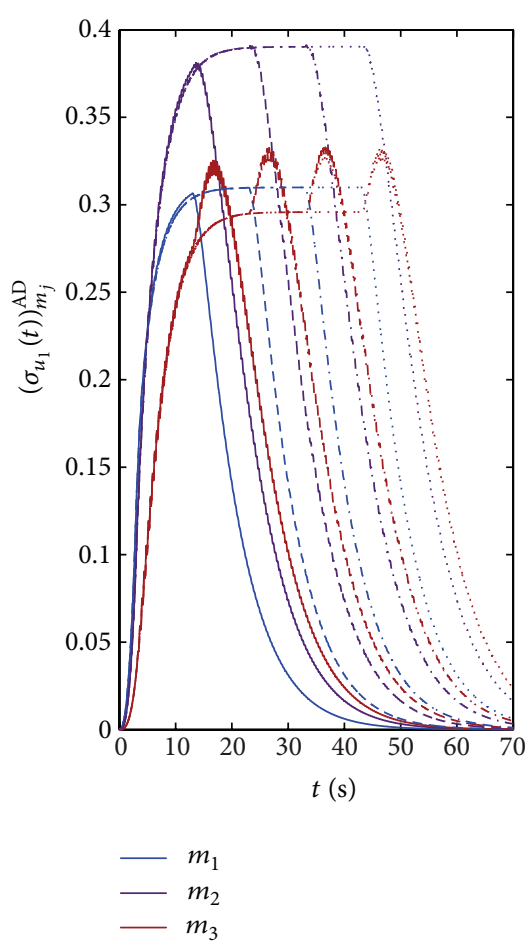

(a)

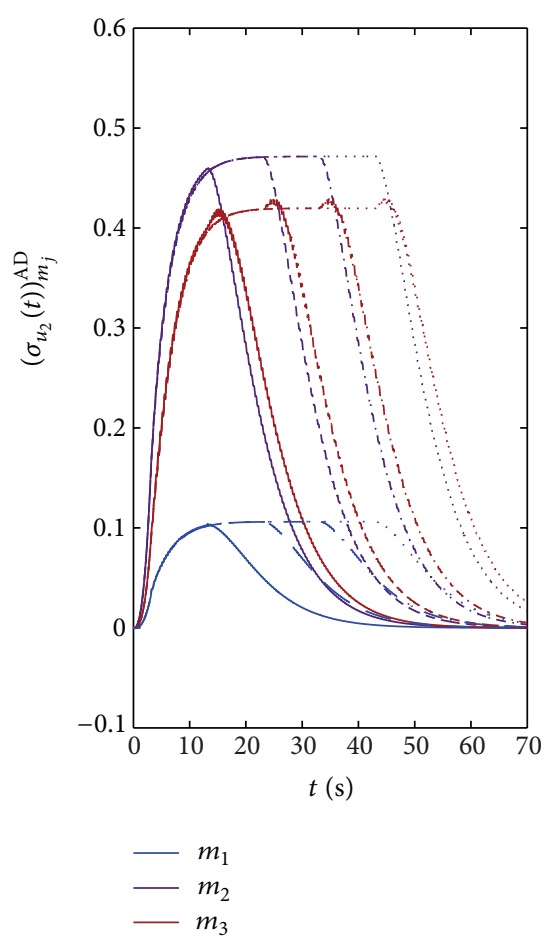

(b)

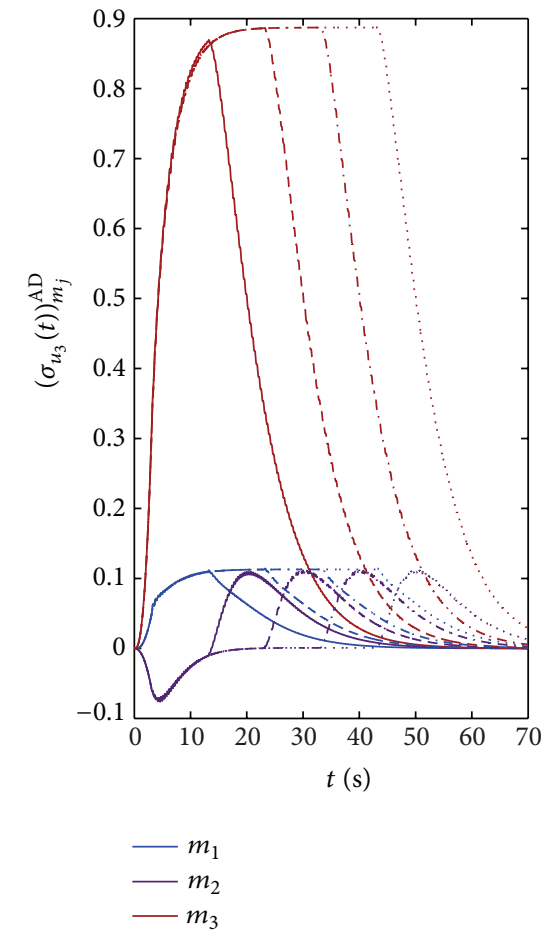

(c)

Figure 5: Covariance displacement sensitivity of a 3-DoF subject to different seismic duration (different line-types, continuous for $t_{d}=10$, lines for $t_{d}=20$, dot-line for $t_{d}=30$, and dotted for $t_{d}=40$ ) with respect to structural masses (different line colours for each storey mass) evaluated at each interstorey drift ((a) for the first, (b) for the second, and (c) for the third). 

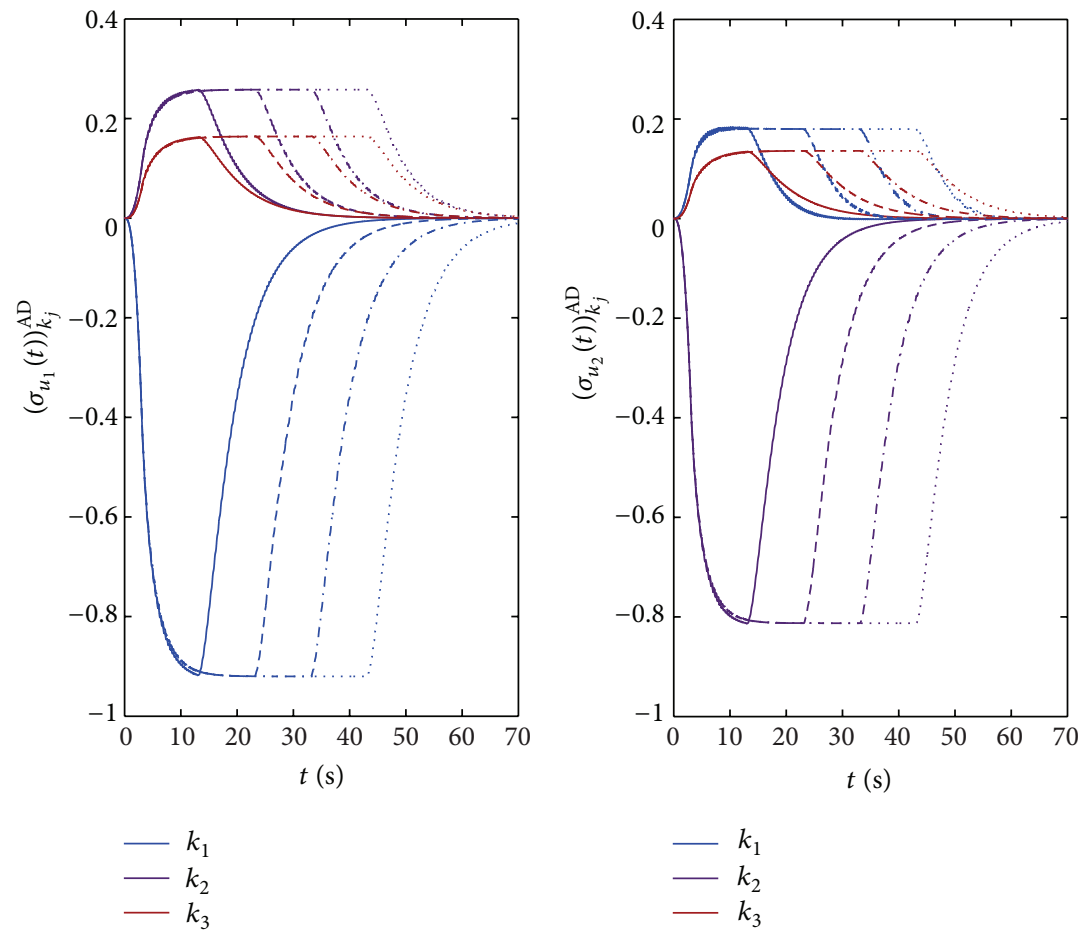

(a)

(b)

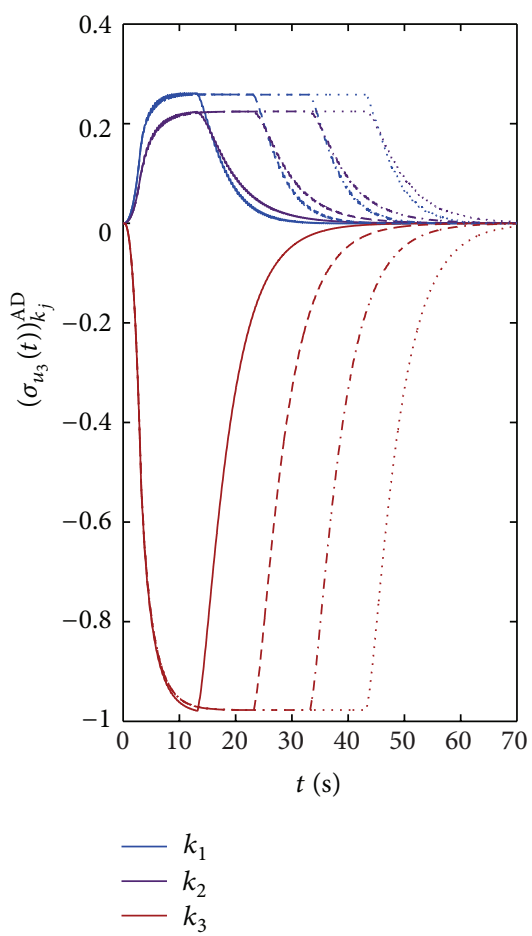

(c)

Figure 6: Covariance displacement sensitivity of a 3-DoF subject to different seismic duration (different line-types, continuous for $t_{d}=10$, lines for $t_{d}=20$, dot-line for $t_{d}=30$, and dotted for $t_{d}=40$ ) with respect to structural stiffness (different line colours for each storey $k_{i}$ ) evaluated at each interstorey drift ((a) for the first, (b) for the second, and (c) for the third). 


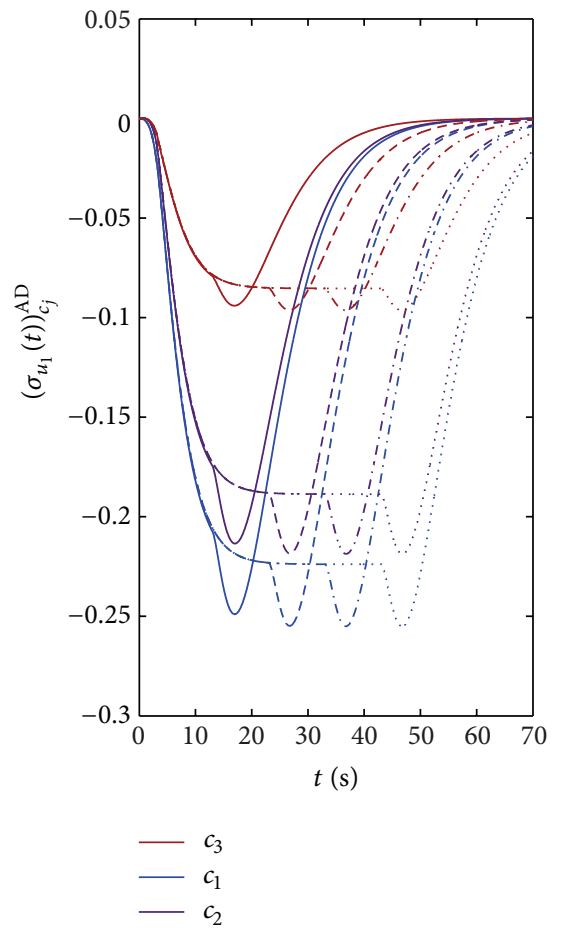

(a)

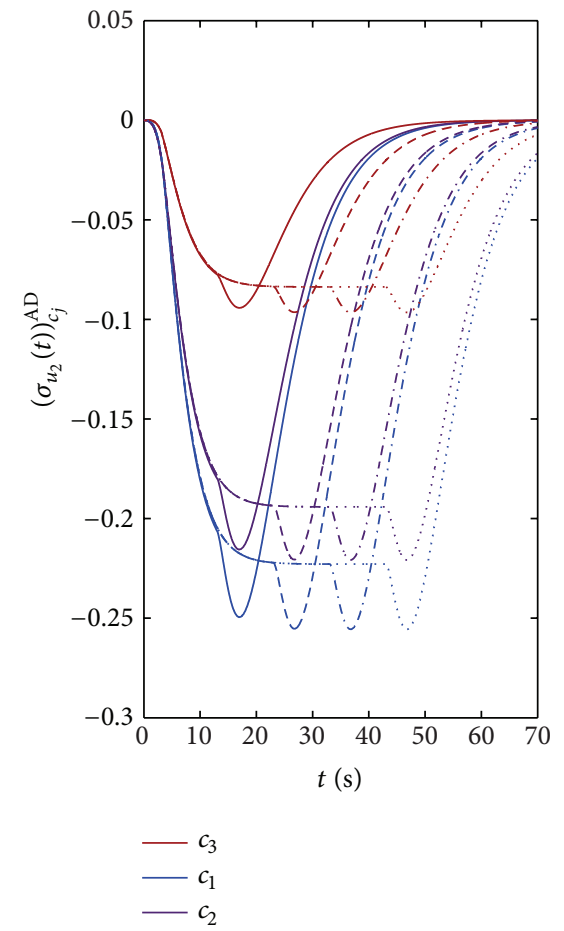

(b)

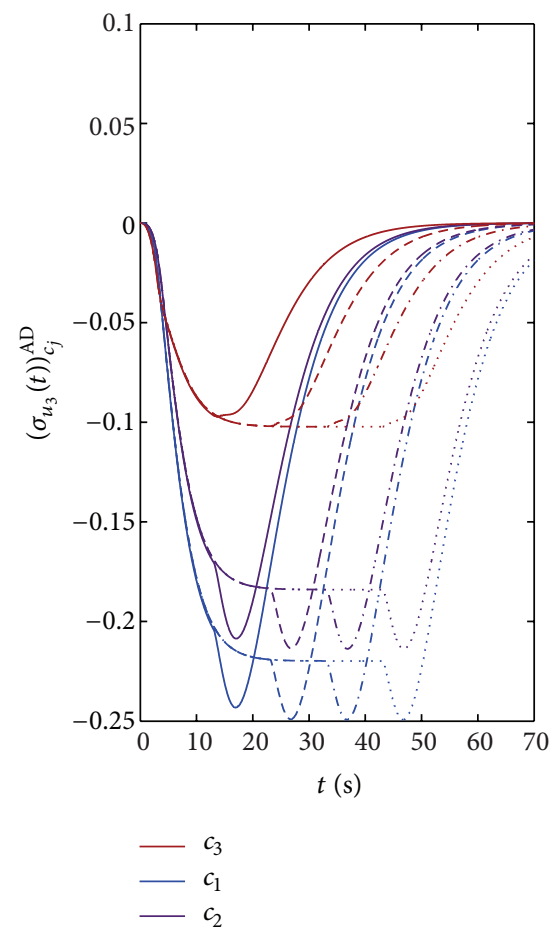

(c)

FIGURE 7: Covariance displacement sensitivity of a 3-DoF subject to different seismic duration (different line-types, continuous for $t_{d}=10$, lines for $t_{d}=20$, dot-line for $t_{d}=30$, and dotted for $t_{d}=40$ ) with respect to structural damping (different line colours for each storey $c_{i}$ ) evaluated at each interstorey drift ((a) for the first, (b) for the second, and (c) for the third). 


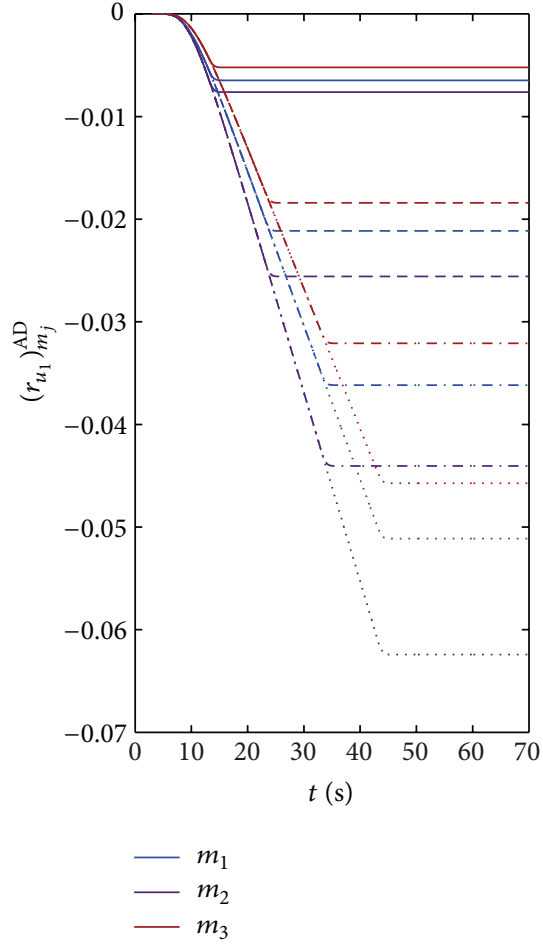

(a)

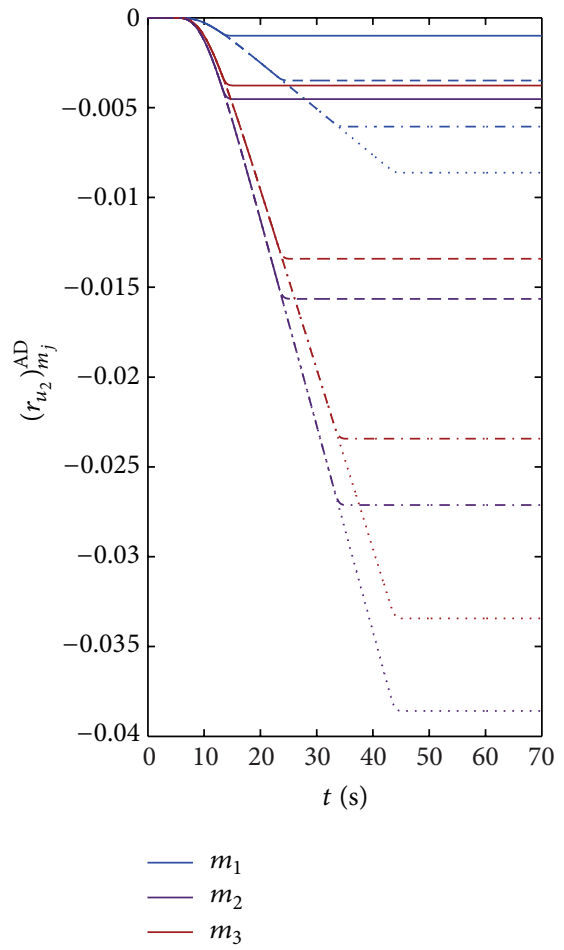

(b)

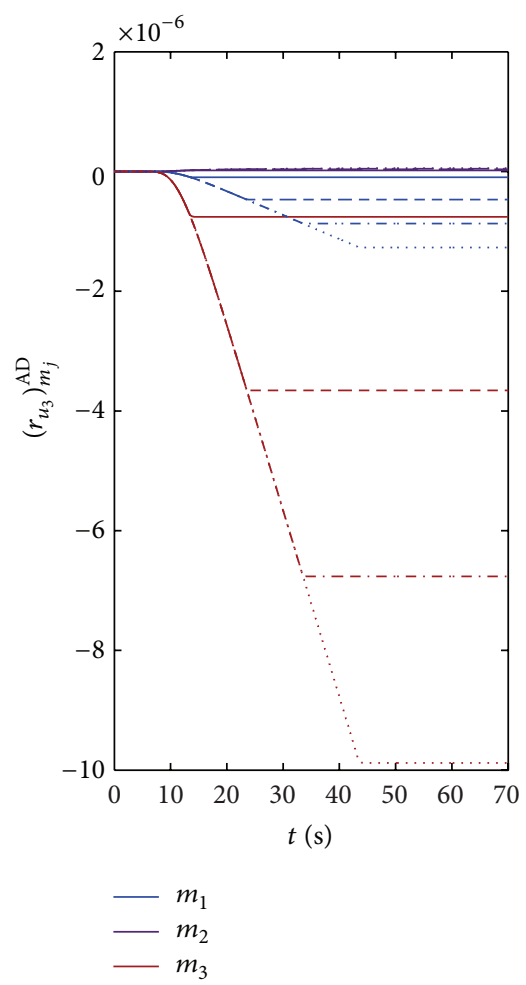

(c)

FIGURE 8: Level reliability $r_{i}$ sensitivity of a 3-DoF subject to different seismic duration (different line-types, continuous for $t_{d}=10$, lines for $t_{d}=20$, dot-line for $t_{d}=30$, and dotted for $t_{d}=40$ ) with respect to structural masses (different line colours for each storey $m_{i}$ ) evaluated at each interstorey drift ((a) for the first, (b) for the second, and (c) for the third). 

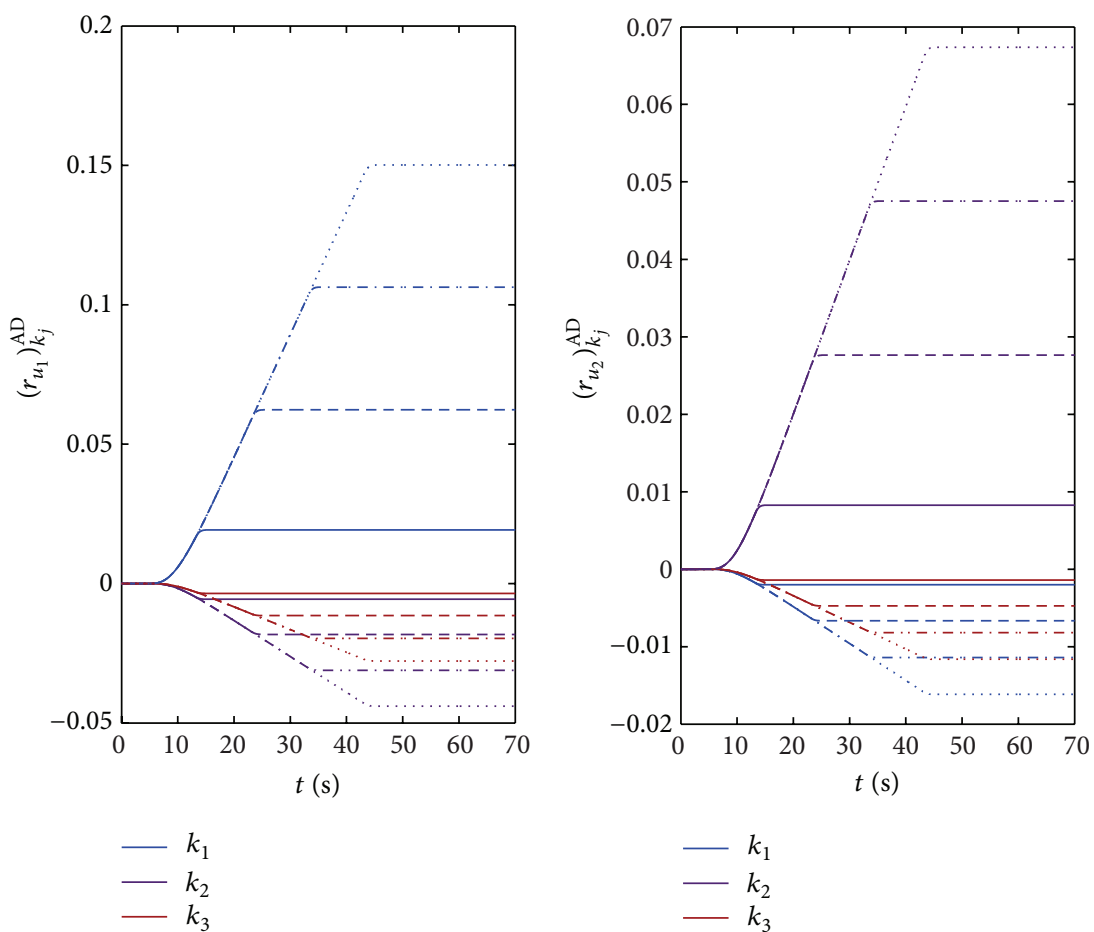

(a)

(b)

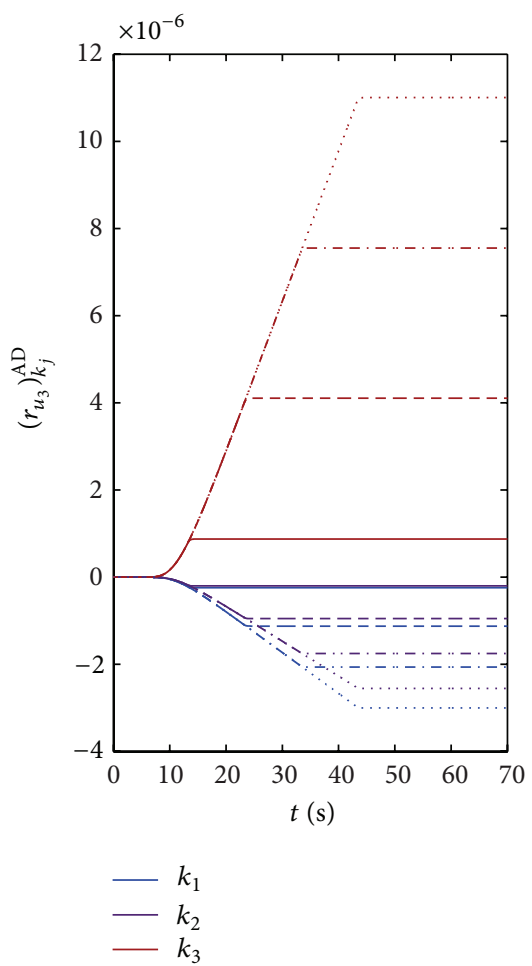

(c)

FIGURE 9: Level reliability $r_{i}$ sensitivity of a 3-DoF subject to different seismic duration (different line-types, continuous for $t_{d}=10$, lines for $t_{d}=20$, dot-line for $t_{d}=30$, and dotted for $t_{d}=40$ ) with respect to structural stiffness (different line colours for each storey $k_{i}$ ) evaluated at each interstorey $\operatorname{drift}$ ((a) for the first, (b) for the second, and (c) for the third). 

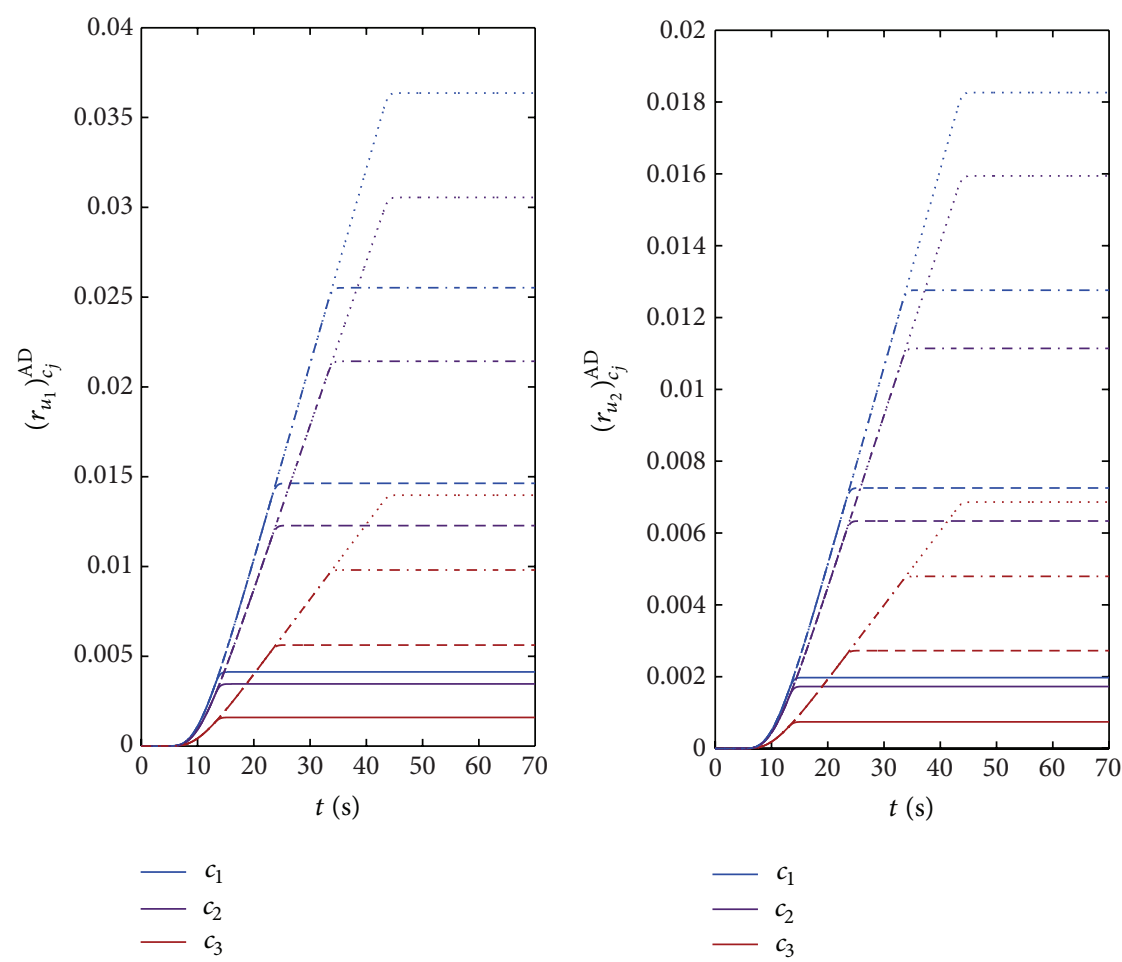

(a)

(b)

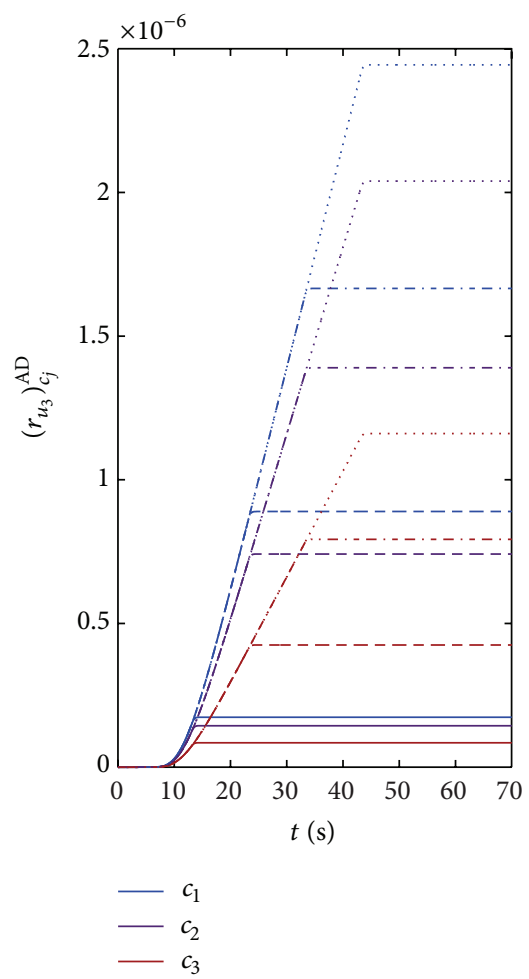

(c)

FIGURE 10: Level reliability $r_{i}$ sensitivity of a 3-DoF subject to different seismic duration (different line-types, continuous for $t_{d}=10$, lines for $t_{d}=20$, dot-line for $t_{d}=30$, and dotted for $t_{d}=40$ ) with respect to structural damping (different line colours for each storey $c_{i}$ ) evaluated at each interstorey drift ((a) for the first, (b) for the second, and (c) for the third). 


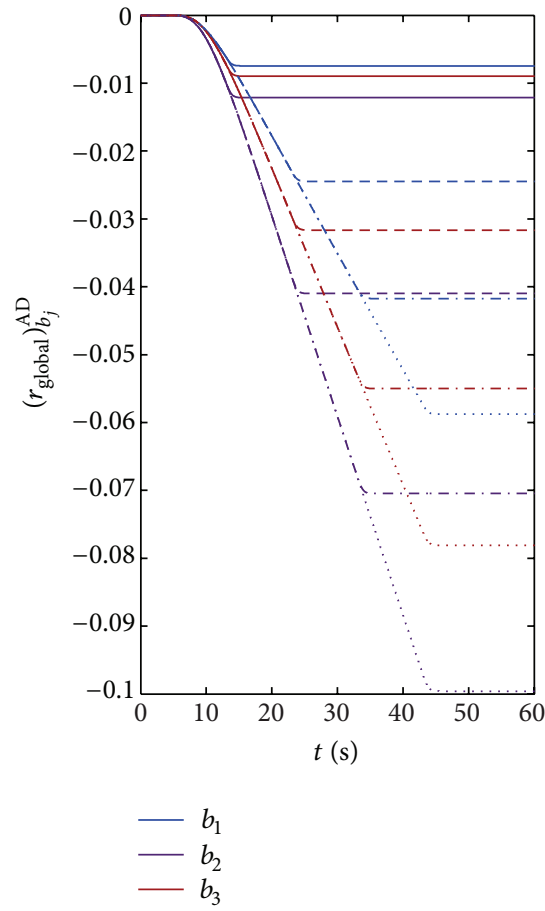

(a)

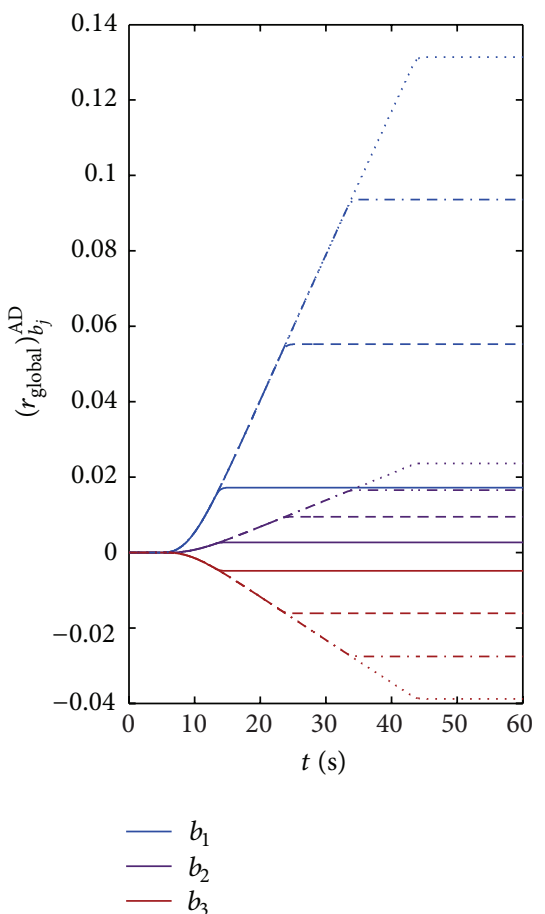

(b)

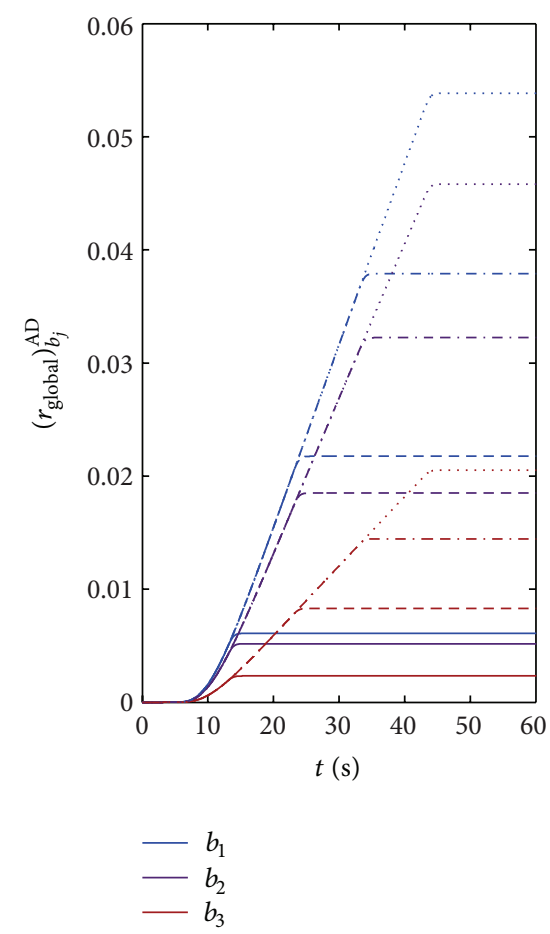

(c)

FIGURE 11: Global reliability sensitivity of a 3-DoF system subject to different seismic duration (different line-types, continuous for $t_{d}=10$, lines for $t_{d}=20$, dot-line for $t_{d}=30$, and dotted for $\left.t_{d}=40\right)$ with respect to structural masses (a), structural stiffness (b), and structural damping (c). 
than that of the analyzed interstorey drift produces a limited response variation.

A different sensitivity of interstorey drifts could be noticed in analysing the effect of stiffness variations, as shown in Figures 6(a), 6(b), and 6(c). It is evident that at each stiffness variation, a reduction of the interiorly variance $\sigma_{u_{i}}$ of the $i^{\text {st }}$-level occurs only when the stiffness $k_{i}$ is increased (blue for $k_{1}$, magenta for $k_{2}$, and red for $k_{3}$ ). On the contrary, for each $i$-level, the increase of a rigidity $k_{j}(j \neq i)$ in levels different from those investigated has an opposite effect, by increasing the lateral displacement variance $\sigma_{u_{i}}$. For example, in Figure 6(b), it should be noticed that $\sigma_{u_{2}}$ decreases when $k_{2}$ increase (magenta line) and meanwhile grows up (with slow velocity) when $k_{1}$ (blue) and $k_{3}$ (red) are increased.

A further effect on $\sigma_{u_{i}}$ has the variation of interstorey dampings. It is evident in Figures $7(\mathrm{a}), 7(\mathrm{~b})$, and $7(\mathrm{c})$ that increasing each of them (blue for $c_{1}$, magenta for $c_{2}$, and red for $c_{3}$ ) has the effect of inducing a reduction in all floors lateral displacement. This is a direct effect of the energy dissipation that is induced by increasing structural damping. Then, the growing up of dissipated energy induces in any case the reduction of elastic and kinetic one, and, therefore, of elastic deformations. With regards to this specific structural system, maximum sensitivities in $\left(\sigma_{u_{i}}\right)_{, b_{j}}^{\mathrm{AD}}$ have about the same magnitude (0.4-1.0) in case of masses and stiffness variations, and a smaller one (0.20-0.25) for damping variations.

First order sensitivities of reliability associated with interstorey drift exceeding over a given barrier, evaluated by (64), are shown in Figures 8, 9, and 10. Also in this case, they are evaluated for different time durations and storey levels, with the same legend structure of previous figures. Results are plotted by using the non-dimensional measure represented by

$$
\left(r_{u_{i}}(t)\right)_{, b_{j}}^{\mathrm{AD}}=\frac{\partial r_{u_{i}}(t)}{\partial b_{j}} \frac{b_{j}}{r_{u_{i}}(t)} .
$$

As in Figures 5, 6, and 7, also in this case, the sensitivities are evaluated with regards to the three floor masses, stiffnesses, and dampings.

Variations are evaluated assuming that $b_{j}=m_{j}$ in Figures 8(a), 8(b), and 8(c), $b_{j}=k_{j}$ in Figures 9(a), 9(b), and 9(c), and finally $b_{j}=c_{j}$ in Figures 10(a), 10(b), and 10(c). Also in these figures Blue lines are for $j=1$, magenta lines are for $j=2$, and red lines are for $j=3$.

Keeping in mind that positive sensitivities indicate an increase of survival probability, it is evident that the above sensitivities have the same trend of lateral drift covariance shown in Figure 3. Besides this, it has to be observed that the reliability sensitivities of the first two floors have comparable values (Figures 8(a), 9(a), and 10(a) and Figures 8(b), 9(b), and $10(\mathrm{~b})$ ), while the sensitivity of $r_{3}$, regarding the top floor, (Figures 8(c), 9(c) and 10(c)) is smaller. This is related to the circumstance that $r_{3}$ practically has a unitary value, and so its variation due to changing in structural parameters is practically negligible.

Finally in Figure 11, the first order sensitivities of global reliability are reported. These are evaluated with respect to variations in structural masses in Figure 11(a), stiffiness in Figure 11(b), and dampings in Figure 11(c). It should be noticed that the global reliability has almost the same behaviour of $r_{1}$ in Figures 8, 9, and 10, being it the smallest one compared with the other two.

\section{Conclusions}

A new method for evaluating the gradient with respect to structural parameters of stochastic response and structural reliability of a multi-degree-of-freedom system subject to nonstationary random loads has been proposed. The random input is modelled by a nonstationary modulated filtered white noise process, able to represent many real physic loads. In order to obtain a general nonstationary approach to be used in different contexts, the structural response is evaluated by a covariance approach, and the time domain differential matrix equations governing the sensitivity of the stochastic response are derived. In particular, the Lyapunov equation is differentiated with respect to the assigned design variables, which could be assumed as structural or input parameters. Based on this result, the structural reliability is also determined. This is defined as the first passage failure probability, expressed by the standard nonstationary expression based on Poissonian first crossing events. Sensitivity of covariance and reliability are then obtained. The proposed formulation computes the sensitivity gradient of covariance matrix and structural reliability with respect to the structural design parameters. A time integration algorithm to solve this problem is proposed.

Finally, a multistorey building idealized by a shear frame structure is considered to study the sensitivity of dynamic responses to a systemic action. The rigid floors masses and interstorey lateral stiffness and damping are considered as design variables. The structural behavior after a modification of these significant parameters has been evaluated by means of non-dimensional sensitivity parameters of covariance response and structural reliability.

\section{Appendices}

\section{A. Time Integration Algorithm}

Even if many numerical standard codes exist for the stationary Lyapunov equation $A X+X A^{T}+B=0$, there are few examples of solution of (21). A simple numeric implicit integration method is then here proposed. This is a modified Euler method in which the time period $[0, T]$ is divided in $m$ equal subperiods $\Delta t=t^{(h+1)}-t^{(h)}(h=0,1, \ldots, m-1)$ and in each subperiod $\Delta t$ a linear variation of the time derivative covariance matrix $\dot{\mathbf{R}}(t)$ is assumed (standard implicit Euler method). Under this assumption, we have

$$
\mathbf{R}^{(h+1)}=\mathbf{R}^{(h)}+\frac{1}{2} \Delta t\left[\dot{\mathbf{R}}^{(h+1)}+\dot{\mathbf{R}}^{(h)}\right]
$$

where the symbol $a^{(h)}$ denotes the generic quantity $a$ evaluated at time $t=h \Delta t$. By using the matrix equations (17) 
evaluated at times $t^{(h+1)}$ and $t^{(h)}$, we get the following $m$ algebraic matrix Lyapunov type equations:

$$
\begin{aligned}
{\left[\left(\frac{1}{2}(\mathbf{I}\right.\right.} & \left.-\Delta t \mathbf{A})) \mathbf{R}^{(h+1)}+\mathbf{R}^{(h+1)}\left(\frac{1}{2}(\mathbf{I}-\Delta t \mathbf{A})\right)^{T}\right] \\
= & {\left[\left(\frac{1}{2}(\mathbf{I}+\Delta t \mathbf{A})\right) \mathbf{R}^{(h)}+\mathbf{R}^{(h)}\left(\frac{1}{2}(\mathbf{I}+\Delta t \mathbf{A})\right)^{T}\right] } \\
& +\frac{\Delta t}{2}\left(\mathbf{B}^{(h)}+\mathbf{B}^{(h+1)}\right),
\end{aligned}
$$

which are solved in sequence for each time value $t^{(h)}$, starting from the initial time value $t^{(0)}=0$ and from the initial covariance matrix value $\mathbf{R}^{(0)}=\mathbf{0}$.

In this way, the $m$ unknown matrices $\mathbf{R}^{(h)},(h=1, \ldots, m)$ are determined. By assuming the matrices as constant or time variable (depending on filter parameters variation)

$$
\begin{aligned}
& \mathbf{P}_{B}=\frac{1}{2}(\mathbf{I}-\Delta t \mathbf{A}), \\
& \mathbf{P}_{F}=\frac{1}{2}(\mathbf{I}+\Delta t \mathbf{A}),
\end{aligned}
$$

we have a more compact form of (A.2):

$$
\begin{aligned}
& {\left[\mathbf{P}_{B} \mathbf{R}^{(h+1)}+\mathbf{R}^{(h+1)} \mathbf{P}_{B}^{T}\right]} \\
& \quad=\left[\mathbf{P}_{F} \mathbf{R}^{(h)}+\mathbf{R}^{(h)} \mathbf{P}_{F}^{T}\right]+\frac{\Delta t}{2}\left(\mathbf{B}^{(h)}+\mathbf{B}^{(h+1)}\right),
\end{aligned}
$$

and each step can be solved by a standard stationary Lyapunov equation solver; for example, the the solver lyap in standard Matlab toolbox,

$$
\mathbf{P}_{B} \mathbf{R}^{(h+1)}+\mathbf{R}^{(h+1)} \mathbf{P}_{B}^{T}+\mathbf{C}^{(h+1)}=0,
$$

where

$$
\mathbf{C}^{(h+1)}=-\left(\left[\mathbf{P}_{F} \mathbf{R}^{(h)}+\mathbf{R}^{(h)} \mathbf{P}_{F}^{T}\right]+\frac{\Delta t}{2}\left(\mathbf{B}^{(h)}+\mathbf{B}^{(h+1)}\right)\right) .
$$

A similar numerical approach could be used for the design sensitivity analysis of the covariance matrix $\mathbf{R}(\bar{b}, t)$. The matrix equations (A.4) are differentiated with respect to design variables $b_{j}$ to, obtain

$$
\begin{aligned}
{\left[\mathbf{P}_{B} \mathbf{R}_{, b_{j}}^{(h+1)}+\mathbf{R}_{, b_{j}}^{(h+1)} \mathbf{P}_{B}^{T}\right] } & \\
= & {\left[\mathbf{P}_{F} \mathbf{R}_{, b_{j}}^{(h)}+\mathbf{R}_{, b_{j}}^{(h)} \mathbf{P}_{F}^{T}\right] } \\
& +\frac{\Delta t}{2}\left[\mathbf{A}_{, b_{j}}\left(\mathbf{R}^{(h+1)}+\mathbf{R}^{(h)}\right)+\left(\mathbf{R}^{(h+1)}+\mathbf{R}^{(h)}\right) \mathbf{A}_{, b_{j}}^{T}\right] \\
& +\frac{\Delta t}{2}\left(\mathbf{B}_{, b_{j}}^{(h+1)}+\mathbf{B}_{, b_{j}}^{(h)}\right) .
\end{aligned}
$$

In the case of matrix $\mathbf{B}$ being independent from the design vector, often a realistic assumption, a more compact form of (A.7) is

$$
\left[\mathbf{P}_{B} \mathbf{R}_{, b_{j}}^{(h+1)}+\mathbf{R}_{, b_{j}}^{(h+1)} \mathbf{P}_{B}^{T}\right]=+\left[\mathbf{P}_{F} \mathbf{R}_{, b_{j}}^{(h)}+\mathbf{R}_{, b_{j}}^{(h)} \mathbf{P}_{F}^{T}\right]+\mathbf{M}^{(h+1)},
$$

where $\mathbf{M}^{(h+1)}=+(\Delta t / 2)\left[\mathbf{A}_{, b_{j}}\left(\mathbf{R}^{(h+1)}+\mathbf{R}^{(h)}\right)+\left(\mathbf{R}^{(h+1)}+\right.\right.$ $\left.\mathbf{R}^{(h)}\right) \mathbf{A}_{, b_{j}}^{T}$ ] and can be solved by a standard stationary Lyapunov solver in the form

$$
\mathbf{P}_{B} \mathbf{R}_{, b_{j}}^{(h+1)}+\mathbf{R}_{, b_{j}}^{(h+1)} \mathbf{P}_{B}^{T}+\mathbf{C}^{(h+1)}=\mathbf{0},
$$

where

$$
\mathbf{C}^{(h+1)}=-\left(\left[\mathbf{P}_{F} \mathbf{R}_{, b_{j}}^{(h)}+\mathbf{R}_{, b_{j}}^{(h)} \mathbf{P}_{F}^{T}\right]+\mathbf{M}^{(h+1)}\right) .
$$

Then, for each design variable $b_{j}$, a sequence of $m$ Lyapunov type matrix algebraic equations are solved in sequence, starting from the initial time $t^{(0)}=0$ and from the initial values $\mathbf{R}_{, b_{j}}^{(0)}=\mathbf{0}$. In this way, for each time $t^{(h)}$ and for each design variable $b_{j}=[\bar{b}]_{j}$, the unknown design sensitivities $\mathbf{R}_{, b_{j}\left(h=1, \ldots, n_{t} ; j=1, \ldots, n_{b}\right)}^{(h)}$ are determined (Algorithm 1).

\section{B. Derivatives of Structural Reliability}

The first order sensitivities of the quantities reported in (29) and (30) are

$$
\begin{aligned}
& \left\{a^{1}(b, t)\right\}_{, b_{j}}=\frac{\left\{\sigma_{\dot{S}}(b, t)\right\}_{, b_{j}}}{\sigma_{S}(b, t)}-\frac{\left\{\sigma_{S}(b, t)\right\}_{, b_{j}} \sigma_{\dot{S}}(b, t)}{\sigma_{S}^{2}(b, t)}, \\
& \left\{a^{2}(b, t)\right\}_{, b_{j}}=-\frac{\rho_{S \dot{S}}(b, t)\left\{\rho_{S \dot{S}}(b, t)\right\}_{, b_{j}}}{\sqrt{1-\rho_{S \dot{S}}^{2}(b, t)}}, \\
& \left\{a^{3}(b, t)\right\}_{, b_{j}}=\frac{\beta\left\{\sigma_{S}(b, t)\right\}_{, b_{j}}}{\sigma_{S}^{3}(b, t)} \exp \left\{-\frac{1}{2}\left(\frac{\beta}{\sigma_{S}(b, t)}\right)^{2}\right\} \text {, } \\
& \left\{\chi\left[d_{S}(b, t)\right]\right\}_{, b_{j}}=\left(-e^{-(1 / 2) d_{x}(b, t)^{2}} d_{x}(b, t)\right. \\
& \left.+\sqrt{2 \pi}\left[1-\frac{1+\operatorname{erf}\left(d_{x}(b, t) / \sqrt{2}\right)}{2 \sqrt{2 \pi}}\right]\right) \\
& \times\left\{d_{x}(b, t)\right\}_{, b_{j}}, \\
& \left\{d_{S}(b, t)\right\}_{, b_{j}}=\left(\beta \left[\sigma_{S}(b, t)\left\{\rho_{S \dot{S}}(b, t)\right\}_{, b_{j}}+\rho_{S \dot{S}}(b, t)\right.\right. \\
& \left.\left.\times\left(1-\rho_{S \dot{S}}^{2}(b, t)\right)\left\{\sigma_{S}(b, t)\right\}_{, b_{j}}\right]\right) \\
& \times\left(\sigma_{S}^{2}(b, t)\left(1-\rho_{S \dot{S}}^{2}(b, t)\right)^{3 / 2}\right)^{-1} .
\end{aligned}
$$

Furthermore, the following relations have been used in determining reliability sensitivities

$$
\left\{\sigma_{S_{i}}(b, t)\right\}_{, b_{j}}=\frac{1}{2} \frac{\left\{\sigma_{S_{i}}^{2}(b, t)\right\}_{, b_{j}}}{\sigma_{S_{i}}(b, t)},
$$




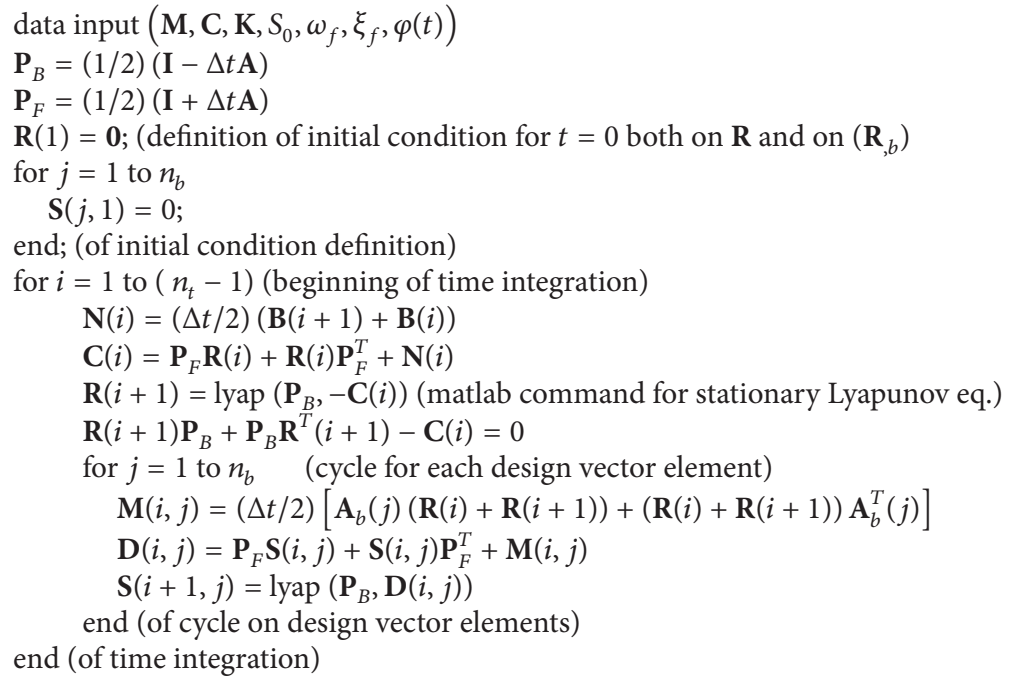

Algorithm 1

$$
\begin{aligned}
&\left\{\sigma_{\dot{S}_{i}}(b, t)\right\}_{, b_{j}}=\frac{1}{2} \frac{\left\{\sigma_{\dot{S}_{i}}^{2}(b, t)\right\}_{, b_{j}}}{\sigma_{\dot{S}_{i}}(b, t)}, \\
&\left\{\rho_{S_{i} \dot{S}_{i}}(b, t)\right\}_{, b_{j}}= \frac{1}{\sigma_{S_{i}}(b, t) \sigma_{\dot{S}_{i}}(b, t)} \\
& \times\left[\left\{\gamma_{S_{i} \dot{S}_{i}}(b, t)\right\}_{, b_{j}}-\gamma_{S_{i} \dot{S}_{i}}(b, t)\right. \\
&\left.\times\left(\frac{\left\{\sigma_{S_{i}}(b, t)\right\}_{, b_{j}}}{\sigma_{S_{i}}(b, t)}+\frac{\left\{\sigma_{\dot{S}_{i}}(b, t)\right\}_{, b_{j}}}{\sigma_{\dot{S}_{i}}(b, t)}\right)\right],
\end{aligned}
$$

where the relations with matrices $\mathbf{R}_{Z_{S} Z_{S}}(b, t)$ and $\left\{\left[\mathbf{R}_{Z_{S} Z_{S}}(b, t)\right]_{i, i}\right\}_{, b_{j}}$ are

$$
\begin{gathered}
\sigma_{S_{i}}^{2}(b, t)=\left[\mathbf{R}_{Z_{S} Z_{S}}(b, t)\right]_{i, i}, \\
\sigma_{\dot{S}_{i}}^{2}(b, t)=\left[\mathbf{R}_{Z_{S} Z_{S}}(b, t)\right]_{n_{s}+i, n_{s}+i}, \\
\gamma_{S_{i} \dot{S}_{i}}(b, t)=\left[\mathbf{R}_{Z_{S} Z_{S}}(b, t)\right]_{i, n_{s}+i}, \\
\left\{\sigma_{S_{i}}^{2}(b, t)\right\}_{, b_{j}}=\left\{\left[\mathbf{R}_{Z_{S} Z_{S}}(b, t)\right]_{i, i}\right\}_{, b_{j}}, \\
\left\{\sigma_{\dot{S}_{i}}^{2}(b, t)\right\}_{, b_{j}}=\left\{\left[\mathbf{R}_{Z_{S} Z_{S}}(b, t)\right]_{n_{s}+i, n_{s}+i}\right\}_{, b_{j}}, \\
\left\{\gamma_{S_{i} \dot{S}_{i}}(b, t)\right\}_{, b_{j}}=\left\{\left[\mathbf{R}_{Z_{S} Z_{S}}(b, t)\right]_{i, n_{s}+i}\right\}_{, b_{j}} .
\end{gathered}
$$

\section{Acknowledgment}

The authors are extremely grateful to Professor Giuseppe Carlo Marano for his enthusiastic support in developing and writing this paper.

\section{References}

[1] T. T. Soong and M. Grigoriu, Random Vibration of Mechanical and Structural Systems, Prentice Hall, 1993.

[2] Y. K. Lin, ProbabilisticTheory of Structural Dynamics, Krieger, Huntington, NY, USA, 1967.

[3] N. Wax, Ed., Stochastic Processes, Dover, 1954.

[4] N. C. Nigam and S. Narayanan, Application of Random Vibration, Narosa, 1994.

[5] L. D. Lutes and S. Sarkani, Stochastic Analysis of Structural and Mechanical Vibration, Prentice-Hall, 1997.

[6] O. C. Zienkiewcz and J. S. Campbell, "Shape optimization and sequential linear programming," in Optimum Structural Design, Theory and Application, R. H. Gallaghar and O. C. Zienkiewcz, Eds., pp. 109-126, John Wiley, London, UK, 1977.

[7] W. Shu-Yu, S. Yanbing, and R. H. Gallagher, "Sensitivity analysis in shape optimization of continuum structures," Computers and Structures, vol. 20, no. 5, pp. 855-867, 1985.

[8] H. M. Adelman and R. T. Haftka, "Sensitivity analysis of discrete structural systems," The American Institute of Aeronautics and Astronautics, vol. 24, no. 5, pp. 823-832, 1986.

[9] J. P. Conte, P. K. Vijalapura, and M. Meghella, "Consistent finite element sensitivities in seismic reliability analysis," in Proceedings of the 13th ASCE Engineering Mechanics Division Conference, pp. 13-16, Johns Hopkins University, June 1999.

[10] S. Benfratello, S. Caddemi, and G. Muscolino, "Gaussian and non-Gaussian stochastic sensitivity analysis of discrete structural system," Computers and Structures, vol. 78, no. 1, pp. 425434, 2000 . 
[11] X. Ma, X. Leng, G. Meng, and T. Fang, "Evolutionary earthquake response of uncertain structure with bounded random parameter," Probabilistic Engineering Mechanics, vol. 19, no. 3, pp. 239-246, 2004.

[12] A. Chaudhuri and S. Chakraborty, "Sensitivity evaluation in seismic reliability analysis of structures," Computer Methods in Applied Mechanics and Engineering, vol. 193, no. 1-2, pp. 59-68, 2004.

[13] P. Cacciola, P. Colajanni, and G. Muscolino, "A modal approach for the evaluation of the response sensitivity of structural systems subjected to non-stationary random processes," Computer Methods in Applied Mechanics and Engineering, vol. 194, no. 4244, pp. 4344-4361, 2005.

[14] M. Barbato and J. Conte, "Structural reliability applications of nonstationary spectral characteristics," Journal of Engineering Mechanics, vol. 137, no. 5, pp. 371-382, 2011.

[15] S. O. Rice, "Mathematical analysis of random noise," Bell System Technical Journal, vol. 23 \& 24, 1944-1945.

[16] V. V. Bolotin, Random Vibrations of Elastic Systems, vol. 8, Aspen, 1984.

[17] A. Naess, "A study of linear combination of load effects," Journal of Sound and Vibration, vol. 129, no. 1, pp. 83-98, 1989.

[18] D. Veneziano, M. Grigoriu, and C. A. Cornell, "Vector process models for system reliability," Journal of Engineering Mechanics, vol. 103, no. 3, pp. 441-460, 1977.

[19] O. Ditlevsen, "First outcrossing probability bounds," Journal of Engineering Mechanics, vol. 110, no. 2, pp. 282-292, 1984.

[20] Y. K. Wen and H. C. Chen, "System reliability under time varing loads: I," Journal of Engineering Mechanics, vol. 115, no. 4, pp. 808-823, 1989.

[21] O. Hagen and L. Tvedt, "Vector process out-crossing as parallel system sensitivity measure," Journal of Engineering Mechanics, vol. 117, no. 10, pp. 2201-2220, 1991.

[22] O. Hagen, "Conditional and joint failure surface crossing of stochastic processes," Journal of Engineering Mechanics, vol. 118, no. 9, pp. 1814-1839, 1992.

[23] J. Song and A. Der Kiureghian, "Joint first-passage probability and reliability of systems under stochastic excitation," Journal of Engineering Mechanics, vol. 132, no. 1, pp. 65-77, 2006.

[24] S. H. Crandall and W. D. Mark, Random Vibration in Mechanical Systems, Academic Press, New York, NY, USA, 1963.

[25] P. C. Jennings, "Periodic response of a general yielding structure," Journal of the Engineering Mechanics Division, vol. 90, no. 2, pp. 131-166, 1964. 


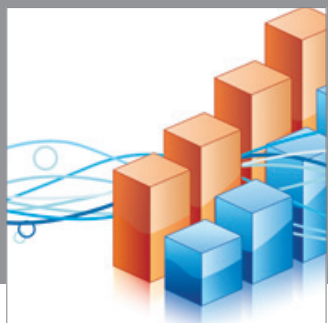

Advances in

Operations Research

mansans

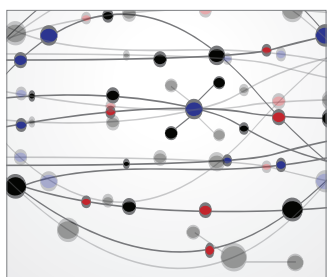

The Scientific World Journal
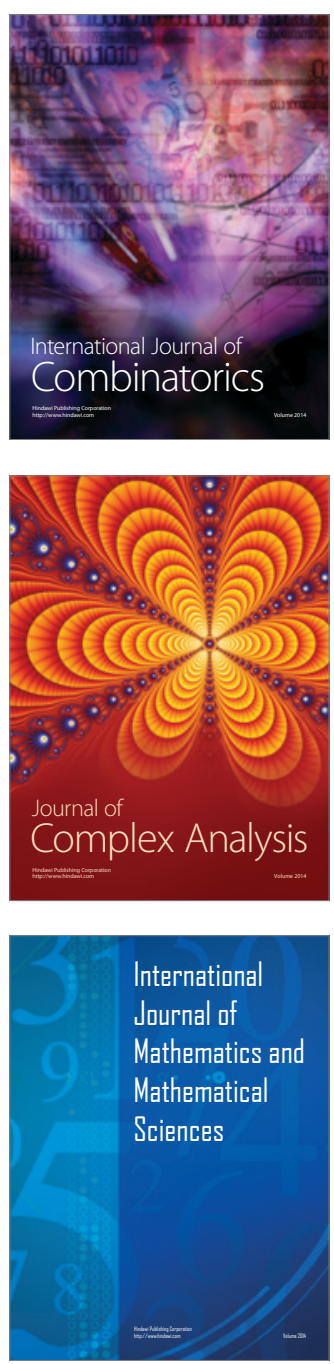
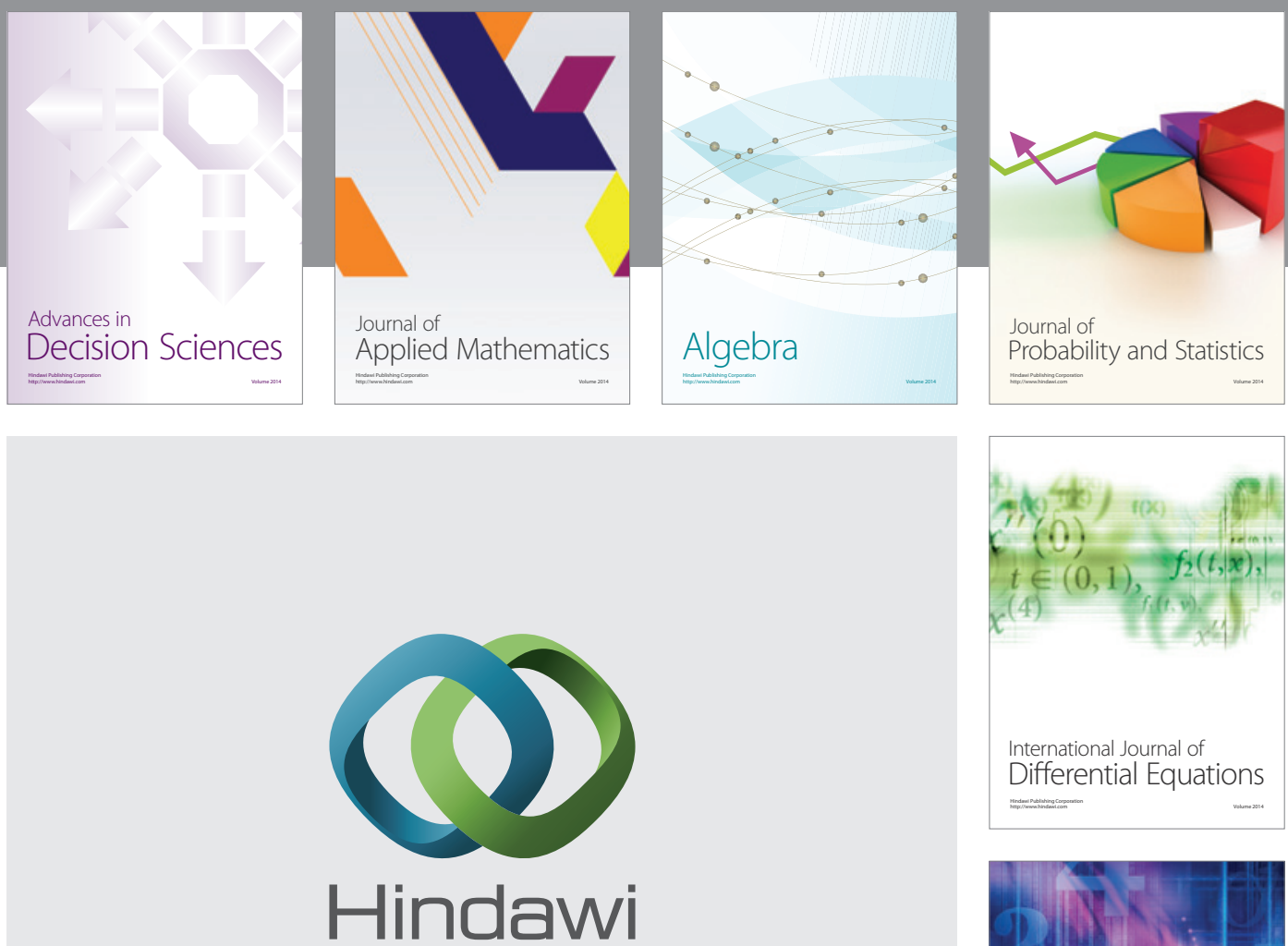

Submit your manuscripts at http://www.hindawi.com
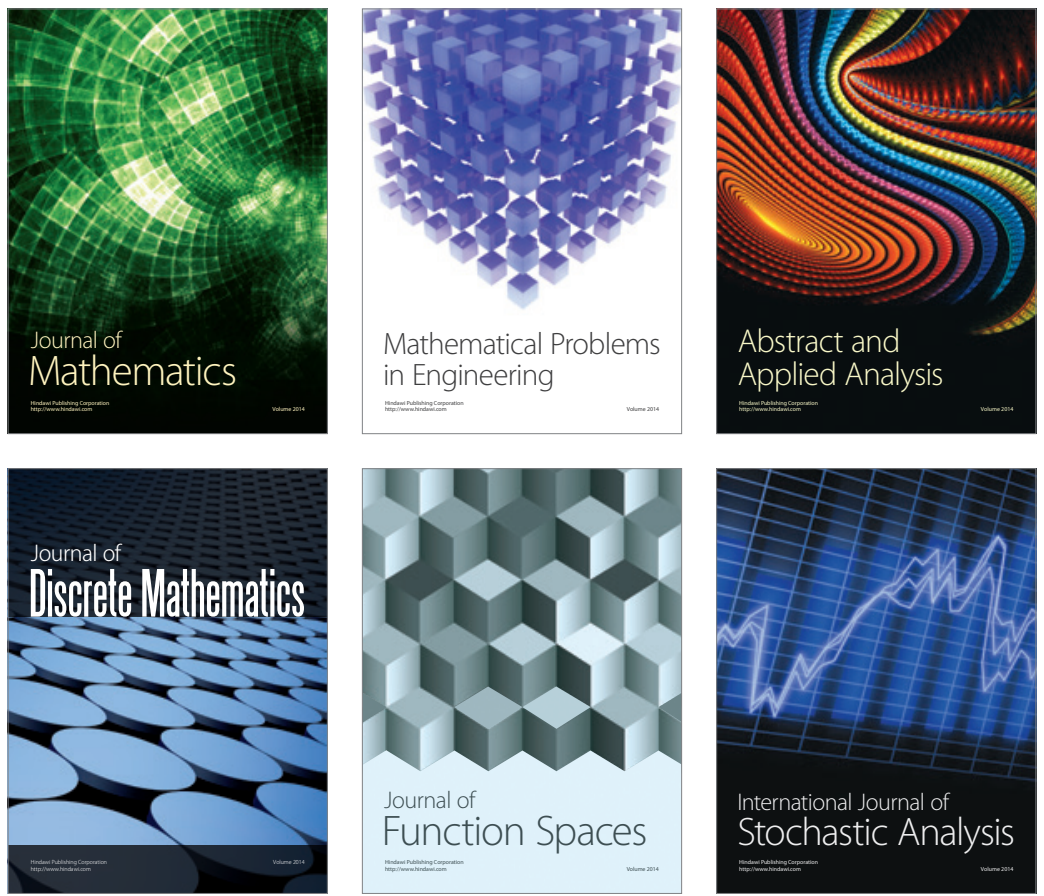

Journal of

Function Spaces

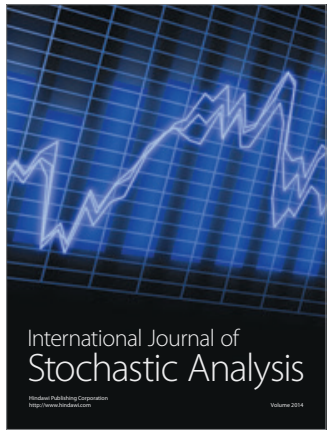

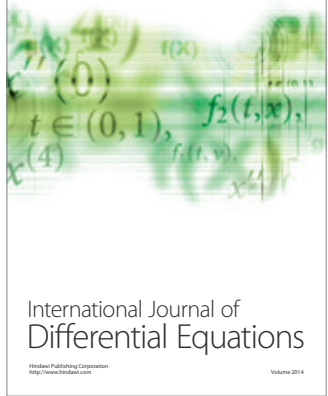
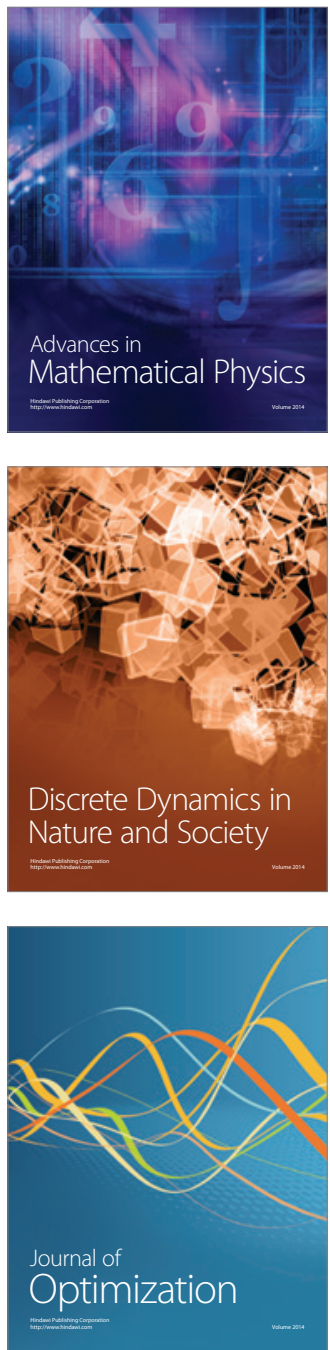